\title{
Estacionariedade em Séries Temporais com Quebras Estruturais
}

\section{Paulo Evandro Dawid}

\author{
Dissertação apresentada \\ ao \\ Instituto de Matemática e Estatística \\ da \\ Universidade de São Paulo \\ para \\ obtenção do grau \\ de \\ Mestre em Estatística
}

Área de concentração: Séries temporais Orientadora: Profa. Dra. Mariane Streibel

Trabalho financiado pelo Programa de Pós-graduação do Banco Central do Brasil.

São Paulo - fevereiro - 2004 


\title{
Estacionariedade em Séries Temporais com Quebras Estruturais
}

\author{
Este exemplar corresponde à redação final \\ da dissertação de mestrado devidamente \\ corrigida e defendida por \\ Paulo Evandro Dawid \\ e aprovada pela comissão julgadora.
}

São Paulo, fevereiro de 2004.

Banca examinadora:

- Profa. Dra. Mariane Streibel (Orientadora) - IME-USP

- Prof. Dr. Pedro Alberto Morettin - IME-USP

- Prof. Dr. Denisard Cnéio de Oliveira Alves - FEA-USP 
Ao meu pai, de quem herdei o prazer pelo conhecimento, e à minha querida mãe. 


\section{Agradecimentos}

Concluída esta etapa, gostaria de agradecer

Ao Banco Central do Brasil que com seu programa de pós-graduação possibilitou e incentivou este trabalho.

À Profa. Mariane Streibel pela orientação e conselhos pertinentes.

Aos professores do IME-USP, sempre disponiveis e fundamentais à minha formação.

À minha esposa Sandra, minha incentivadora cotidiana.

Aos meus pais e à minha família, com os quais sempre posso contar.

Aos amigos do IME e do Banco Central, pelo companheirismo e apoio nesta jornada. 


\section{Resumo}

Um dos principais problemas na análise de séries temporais é testar se uma determinada série é estacionária ou não. Séries estacionárias são, grosso modo, séries que mantêm o mesmo comportamento ao longo do tempo. A teoria e várias técnicas de análise já estão bem estabelecidas para essas séries. A análise de séries não-estacionárias, por sua vez, é mais complexa e não existe uma abordagem geral.

Os testes de raiz unitária são os mais utilizados para se testar um tipo especifico de nãoestacionariedade, denominada tendência estocástica ou passeio aleatório.

Este trabalho pretende explorar uma abordagem alternativa desenvolvida no contexto de modelos de componentes não-observáveis. São os chamados testes de estacionariedade, que diferem dos testes de raiz unitária pela inversão da hipótese nula.

Os testes de estacionariedade vêm apresentando generalizações recentes, possibilitando a aplicação a uma gama mais ampla de séries: com tendência, com sazonalidade e com quebras estruturais.

Nessa linha, pretende-se então revisar os principais conceitos relacionados e aplicar esses testes de estacionariedade a séries econômicas brasileiras.

Palavras-chave: Componentes não-observáveis, distribuição de Cramér-von Mises, estacionariedade, quebras estruturais, tendência estocástica, teste invariante localmente ótimo. 


\section{Abstract}

Testing for stationarity is an important issue in time series analysis. Stationary series are, in a certain way, series that keep their behaviour as time goes by. The theory and many analysis tools are already available for stationary series. The analysis of nonstationary series is more difficult and there is not a general approach.

Unit root tests are the most popular ones to test the specific kind of nonstationarity called stochastic trend or random walk behaviour.

This work intends to explore an alternative approach developed for unobserved components models. The correspondent tests are called stationarity tests and differ from unit root tests by inverting the null hypothesis.

Stationary tests present some recent generalizations for applications in a broader class of series: with trend, sazonality or structural breaks.

Following this new generalizations, we intend to review the main related concepts and use the stationary tests in Brazilian economic data.

Keywords: Unobserved components, Cramér-von Mises distribution, stationarity, structural breaks, stochastic trend, locally best invariant test. 


\section{Sumário}

1 Introdução 1

1.1 Motivação econômica . . . . . . . . . . . . . . . . . . . . . 1

1.2 Motivação estatística . . . . . . . . . . . . . . . 3

1.2 .1 Testes de raiz unitária . . . . . . . . . . . . . . . . . 3

1.2.2 Testes de estacionariedade . . . . . . . . . . . . . . 4

1.3 Plano do trabalho ................... 7

2 Definições e resultados básicos $\quad 9$

2.1 Processos estocásticos e estacionariedade . . . . . . . . . . . . . . . 9

2.2 Movimento browniano e ponte browniana . . . . . . . . . . . . . . 10

2.3 Inversão numérica da função característica . . . . . . . . . . . . . . 11

2.4 Distribuições da família Cramér-von Mises . . . . . . . . . . . . . . 12

2.5 Teste invariante localmente ótimo - ILO . . . . . . . . . . 16

2.5 .1 Teste localmente ótimo . . . . . . . . . . . . . 16

2.5.2 Teste invariante . . . . . . . . . . . . . . . 17

2.5.3 Teste ILO para o modelo linear normal . . . . . . . . . . . 18

3 Variância de longo termo $\quad 20$

3.1 A variância de longo termo $-\sigma_{L}^{2} \ldots \ldots . . \ldots . . . . . . . .20$

3.2 Metodologia proposta para a estimação de $\sigma_{L}^{2} \ldots \ldots . . . . . . .26$

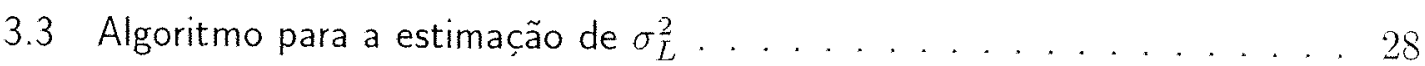


4 Testes de estacionariedade $\quad 30$

4.1 Série com nível constante . . . . . . . . . . . . . . . . . . 31

4.2 Série com tendência linear . . . . . . . . . . . . . . . . . 32

4.3 Série com quebra no nível e na tendência . . . . . . . . . . . 33

4.4 Série com quebra no nível apenas . . . . . . . . . . . . . 35

4.5 Extensão para ruídos estacionários . . . . . . . . . . . . . . . . . . 36

4.6 Modelos com componente sazonal . . . . . . . . . . . . . . . 37

5 Simulações 38

5.1 Estimativa da variância de longo termo . . . . . . . . . . . . . 38

5.2 Tamanho e poder para amostras finitas . . . . . . . . . . . . 40

5.3 Tamanho e poder do teste simplificado . . . . . . . . . . . . . . 43

6 Aplicações 47

6.1 Análise das exportações brasileiras . . . . . . . . . . . . . . . . . . 47

6.2 Análise das importações brasileiras . . . . . . . . . . . . . . 53

6.3 Câmbio real brasileiro ...................... 61

$\begin{array}{lc}\text { Referências Bibliográficas } & 63\end{array}$ 


\section{Capítulo 1}

\section{Introdução}

\subsection{Motivação econômica}

A não-estacionariedade é uma característica comum em muitas séries temporais financeiras e econômicas. Agregados macroeconômicos, como o PIB, a produção industrial, o investimento etc., em geral apresentam tendência de crescimento. Isso reflete a própria dinâmica do desenvolvimento econômico, que por sua vez é explicado em diversas teorias de desenvolvimento por fatores como a acumulação de capital, o nível de poupança e investimento, o crescimento da população economicamente ativa, os ganhos de produtividade advindos da inovação tecnológica etc. Dada essa "tendência natural" ao crescimento, essas séries são passíveis de serem caracterizadas com um componente de tendência determinística.

Já as séries financeiras, como índices de preço de ações, taxas de câmbio em regime livre, preços de commodities etc., usualmente apresentam não-estacionariedade caracterizada por um componente que é um passeio aleatório, ou seja, uma tendência dita estocástica. Esse resultado empírico também pode ser explicado pela dinâmica de formação desses preços e está de acordo com modelos econômicos baseados em expectativas racionais: os agentes desses mercados, quando dotados de todas as informações relevantes dis- 


\subsection{Motivação econômica}

poniveis, conseguem antecipar perdas ou ganhos futuros esperados. $E$ isso reflete no valor atual do ativo, de forma que a série desses preços presentes corresponde a uma série acumulada de ruídos, ou seja, de choques aleatórios imprevisíveis por parte dos agentes econômicos. Esse comportamento é chamado de 'passeio aleatório'.

Quando um componente da série temporal é um passeio aleatório, costuma-se dizer que a série possui uma raiz unitária, ou que é integrada de ordem $1, I(1)$. A relevância de se saber se uma série possui raiz unitária está no fato de que, em caso afirmativo, os choques externos causam um efeito permanente na série. Ao passo que, em uma série estacionária, há um retorno à média após um certo tempo. Por exemplo, se a série de um agregado macroeconômico possuir um componente que é um passeio aleatório, isto é, se sua tendência é estocástica, tem-se como principal consequência empírica que os choques externos significativos, reais ou monetários, terão um efeito permanente nessa série. Assim, as políticas monetárias e fiscais que afetam tal série passam a exigir maior atenção, pois seus efeitos não são transitórios.

Nessa linha, Nelson \& Plosser (1982), num influente trabalho, analisaram 13 séries macroeconômicas dos Estados Unidos, aplicando a elas o teste de Dickey-Fuller (DF) para raiz unitária [v. abaixo]. Como resultado, obtiveram que, para a maioria das séries, não se pode rejeitar a hipótese de que possuem uma raiz unitária. Assim, puseram em questão a maneira tradicional de se modelar as séries macroeconômicas como uma tendência determinística mais um ruído estacionário. E lançaram uma agenda de pesquisa que de certa forma estende-se até hoje, haja vista a importância para a validação de modelos da teoria econômica. Do ponto de vista estatístico, o desafio é obter melhores testes, pois com base em amostras finitas não se pode afirmar com precisão absoluta se uma série temporal possui ou não um componente não observado que é um passeio aleatório, ou raiz unitária. 


\subsection{Motivação estatística}

Para uma visão mais detalhada e abrangente, ver, por exemplo, Maddala \& Kim (1998).

\subsubsection{Testes de raiz unitária}

O teste mais popular para a verificação da não-estacionariedade de uma série é o teste de Dickey-Fuller, ou sua versão aumentada (ADF). Ele consiste basicamente em testar se o modelo auto-regressivo (ou mesmo ARMA) que ajusta a série possui uma raiz unitária no polinômio auto-regressivo $\phi(L)$. Ajusta-se, por exemplo, a série $y_{t}$ por um modelo $\operatorname{AR}(p)$ :

$$
\begin{aligned}
\phi(L) y_{t} & =\varepsilon_{t}, \quad \varepsilon_{t} \sim \mathcal{N} \mathrm{id}\left(0, \sigma^{2}\right) \\
\phi(L) & =1-\phi_{1} L-\phi_{2} L^{2}-\ldots-\phi_{p} L^{p} \\
L y_{t} & =y_{t-1}: \quad L \text { é o operador defasagem } \\
& \mathcal{N} \text { id indica distribuição normal e independente }
\end{aligned}
$$

E testa-se então se o polinômio $\phi(L)$ possui uma raiz no círculo unitário. A distribuição assintótica do estimador, sob a hipótese de raiz unitária, é diferente da t-Student obtida sob a alternativa da série ser estacionária, ou seja, quando as raízes de $\phi(L)$ caem fora do círculo unitário.

Na linha dos testes de raiz unitária, podem-se destacar sofisticações importantes, como - teste de Phillips-Perron ${ }^{1}$ que permite que os erros $\varepsilon_{t}$ possuam uma estrutura de dependência fraca. O que é mais realista no contexto de séries temporais e, em geral, é suficiente para abranger grande parte das séries econômicas reais. Perron (1989) obtém as distribuições assintóticas para séries contendo quebras estruturais, o que flexibiliza e aumenta o poder dos testes de raiz unitária, se tais quebras de fato existem.

\footnotetext{
${ }^{1}$ Phillips (1987) e Phillips \& Perron (1988)
} 


\subsubsection{Testes de estacionariedade}

Numa linha de pesquisa paralela, desenvolveram-se os chamados testes de estacionariedade. Como os testes de raiz unitária, eles também servem para verificar se uma série temporal é estacionária ou não, mas com uma modelagem distinta. Do ponto de vista estatístico, a principal diferença está na inversão da hipótese nula:

- Nos testes de raiz unitária, testa-se:

$\mathbf{H}_{0}$ : a série, condicionalmente ao modelo, possui raiz unitária e, portanto, não é estacionária.

Contra a hipótese alternativa:

$\mathrm{H}_{\mathrm{a}}$ : todas as raízes do modelo estão fora do círculo unitário e, portanto, a série é estacionária.

- Nos testes de estacionariedade, o modelo é a soma de um componente estacionário e um componente não-estacionário (passeio aleatório). Então, testa-se:

$\mathbf{H}_{0}$ : o passeio aleatório não está presente e, portanto, a série é estacionária.

Contra a hipótese alternativa:

$\mathbf{H}_{\mathrm{a}}$ : o passeio aleatório está presente e, portanto, a série não é estacionária.

Simplificadamente, o modelo para o teste de estacionariedade pode ser esquematizado como:

$$
\begin{aligned}
& y_{t}=\mu_{t}+\varepsilon_{t}, \quad \varepsilon_{t} \sim \mathcal{E S T}\left(0, \gamma_{\varepsilon}\right) \\
& \mu_{t}=\mu_{t-1}+\eta_{t}, \quad \eta_{t} \sim \operatorname{RB}\left(0, \sigma_{\eta}^{2}\right) \\
& \varepsilon_{t} \quad \mathrm{e} \quad \eta_{t} \text { independentes entre si. }
\end{aligned}
$$

E o teste de hipóteses correspondente seria:

- $H_{0}: \sigma_{\eta}^{2}=0$

- $H_{a}: \sigma_{\eta}^{2}>0$ 


\subsection{Motivação estatística}

\section{Um pouco de história}

Em termos de aperfeiçoamento, os testes de estacionariedade seguiram mais ou menos a mesma trajetória do testes de raiz unitária. Com base no modelo (1.2), relata-se a seguir as principais evoluções dos testes de estacionariedade.

Nyblom \& Makelainen (1983) desenvolvem um teste (NM) em que $\varepsilon_{t}$ também é um ruído branco e $\varepsilon_{t}$ e $\eta_{t}$ possuem distribuições normais independentes. Nessas condições, NM é o teste invariante localmente ótimo (ILO ou LBI de locally best invariant) e, sob $H_{0}$, tem assintoticamente distribuição de Cramér-von Mises (CVM). A estatística de teste obtida $\xi$ possui a seguinte região crítica:

$$
\xi=\frac{\sum_{i=1}^{T}\left(\sum_{t=1}^{i} e_{t}\right)^{2}}{T^{2} \hat{\sigma}^{2}}>c(\alpha)
$$

Em que $T$ é o tamanho da série, $e_{t}, t=1, \ldots, T$, são os resíduos do modelo ajustado, $c(\alpha)$ é o valor crítico dependente do nível de significância $\alpha$ e $\hat{\sigma}^{2}$ é a variância amostral:

$$
\hat{\sigma}^{2}=\frac{1}{T} \sum_{t=1}^{T}\left(y_{t}-\bar{y}\right)^{2}
$$

A derivação da distribuição assintótica de $\xi$ baseia-se em resultados da teoria de processos estocásticos ${ }^{2}$. Sob $H_{0}$, tem-se que a soma parcial dos resíduos, devidamente ajustada, converge fracamente para a chamada ponte browniana, $B(r)$, definida em termos do processo de Wiener padrão, $W(r)$ :

$$
\frac{1}{\sigma \sqrt{T}} \sum_{i=1}^{[T r]} e_{i} \Rightarrow B(r)=W(r)-r W(1), \quad r \in[0,1]
$$

' $\Rightarrow$ ' indica convergência fraca de medidas de probabilidade associadas. Tomando-se o limite em (1.3), tem-se:

\footnotetext{
${ }^{2}$ v., e.g., Tanaka (1996)
} 


$$
\xi \Rightarrow \int_{0}^{1} B(r)^{2} d r
$$

que corresponde à distribuição de Cramér-von Mises.

Os demais resultados assintóticos obtidos nesses testes de estacionariedade, e mesmo nos testes de raiz unitária, derivam de raciocínios análogos.

Em 1986, Nyblom generaliza o modelo em (1.2) para permitir uma tendência linear:

$$
y_{t}=\mu_{t}+\beta t+\varepsilon_{t}
$$

A estatística utilizada ainda é a mesma $\xi$, mas a distribuição assintótica é uma generalização da CVM, denotada por $\mathrm{CVM}_{2}$.

Kwiatkowski, Phillips, Schmidt \& Shin (1992), analogamente a Phillips \& Perron (1988) no contexto de raiz unitária, generalizam o teste NM de forma que $\varepsilon_{t}$ possa ser um processo estacionário sob certas condições. De novo, uma hipótese mais realista para séries temporais empíricas. A estatística utilizada para esse teste, denominado KPSS, continua a ser a $\xi$ acima, com as mesmas distribuições assintóticas, mas agora substituindo $\hat{\sigma}^{2}$ por um estimador consistente da chamada variância de longo termo da série, $\sigma_{L}^{2}(l)$, ou seja, requer-se que

$$
\lim _{T \rightarrow \infty} \mathbb{P}\left(\left|\hat{\sigma}_{L}^{2}(l)-\sigma_{L}^{2}\right|>\epsilon\right)=0 \quad, \quad \forall \epsilon>0
$$

$l$ indica o tamanho do núcleo $w(t, l)$ utilizada por Kwiatkowski et al. (1992) para obter um estimador consistente não-paramétrico, baseado na teoria espectral de séries temporais ${ }^{3}$. Utilizando uma janela de Bartlett ${ }^{4}$, obtiveram:

$$
\hat{\sigma}_{L}^{2}(l)=\frac{1}{T}\left[\sum_{i=1}^{T} e_{i}^{2}+2 \sum_{t=1}^{l} w(t, l) \sum_{i=t+1}^{T} \epsilon_{t} \epsilon_{i-t}\right]
$$

\footnotetext{
${ }^{3}$ v., e.g., Priestley (1981)

${ }^{4}$ Outras janelas podem ser utilizadas, v, e.g. Andrews (1991)
} 
com

$$
w(t, l)=1-\frac{t}{l+1} \quad, \quad t=1, \ldots, l
$$

Busetti \& Harvey (2001) estenderam os testes NM e KPSS acima para séries contendo quebras estruturais (teste $\mathrm{BH}$ ). Mais especificamente, séries com ou sem tendência linear que possuam descontinuidades (uma ou mais) no nível ou na inclinação. O modelo mais geral, como extensão de (1.2), seria:

$$
y_{t}=\mu_{t}+\beta t+\delta_{\mu} w_{t}+\delta_{\beta}\left(w_{t} t\right)+\varepsilon_{t}
$$

Supondo uma quebra, no nível e na inclinação, no instante $\tau$, tem-se:

$$
w_{t}=\mathbf{1}_{\{t>\tau\}}, \quad t=1, \ldots, T, \quad 1<\tau<T
$$

Busetti \& Harvey (2001) obtiveram as distribuições assintóticas, em termos do funcional $W(r)$, de quatro variações possiveis do modelo:

- Quebra no nível no modelo sem tendência.

- Quebra no nível e na inclinação no mesmo instante $\tau$.

- Quebra apenas no nível no modelo com tendência.

- Quebra apenas na inclinação no modelo com tendência.

\subsection{Plano do trabalho}

No capítulo 2 apresentamos alguns conceitos básicos e resultados. Em particular, a metodologia para obtenção das distribuições da família Cramér-von Mises via inversão da função característica e os conceitos por trás dos testes invariantes e localmente ótimos utilizados. No capítulo 3 introduzimos o conceito de variância de longo termo, sua relação com a função de densidade espectral e propomos uma metodologia para sua estimativa, baseada em estimação espectral não-paramétrica. No capítulo 4 apresentamos 
os modelos com tendência e quebras utilizados, seus respectivos testes de estacionariedade, uma forma simplificada para os testes e algumas extensões: para séries com ruído estacionário em vez de ruído branco e para séries com componentes sazonais nãoaleatórias. No capítulo 5 realizamos algumas simulações para: (i) avaliar a qualidade da estimativa proposta para a variância de longo termo, (ii) avaliar o tamanho e poder empíricos do teste com quebra no nível e na tendência utilizando a estimativa proposta da variância de longo termo e (iii) avaliar em termos de tamanho e poder empíricos a qualidade do teste simplificado. E no capítulo 6, aplicamos alguns dos testes para dados econômicos brasilieiros: exportação, importação e taxa de câmbio real. 


\section{Capítulo 2}

\section{Definições e resultados básicos}

Com o intuito de se fazer uma breve revisão e uma padronização da notação, definiremos neste capítulo alguns conceitos básicos relevantes. Também serão apresentados alguns resultados utilizados no decorrer deste trabalho.

\subsection{Processos estocásticos e estacionariedade}

Definição 2.1 (Processo estocástico). Um processo estocástico é uma famúlia de variáveis aleatórias $\{X(t, \omega), t \in \mathbf{T}, \omega \in \Omega\}$ definidas em um espaço de probabilidade $(\Omega, \mathcal{F}, P)$. Tais variáveis são indexadas pelo conjunto $\mathbf{T}$, que aqui possuirá uma relação de ordem definida: se $t_{1}, t_{2} \in \mathbf{T}$, então $t_{1} \leq t_{2}$ ou $t_{1}>t_{2}$.

Definição 2.2 (Processo normal). Um processo estocástico $\{X(t), t \in \mathbf{T}\}$ é denominado normal se todas as suas distribuições finito-dimensionais são normais multivariadas, ou seja,

$$
\left(X\left(t_{1}\right), X\left(t_{2}\right), \ldots, X\left(t_{n}\right)\right) \sim \mathcal{N}_{n}(\mu, \Sigma), \quad \forall t_{1}, t_{2}, \ldots, t_{n} \in \mathrm{T}
$$

Como os processos a serem analisados são observados em intervalos de tempo regulares, discretos portanto, chamaremos de série temporal a uma realização de um processo estocástico para um conjunto enumerável T. Ou seja, $\left\{y_{t}\right\}$ será uma série temporal se 
$\left\{y_{t}\right\}_{t=1,2, \ldots,|\mathbf{T}|}=\{X(t, \omega): t=1,2, \ldots,|\mathbf{T}| ; \omega \in \Omega ; \omega$ fixo $\}$, para algum processo estocástico $X(t, \omega)$.

Definição 2.3 (Série estacionária). Uma série temporal $\left\{y_{t}\right\}_{t \in \mathbf{T}}$ é denominada fracamente estacionária, ou estacionária de segunda ordem, ou, simplesmente, estacionária, se, para todo $t \in \mathbf{T}$,

1. $\mathrm{E} y_{t}=\mu=c t e$

2. $\operatorname{Var} y_{t}=\sigma^{2}=$ cte $<\infty$

3. $\operatorname{Cov}\left(y_{t}, y_{t+|h|}\right)=\gamma(h), \quad h \in \mathbb{Z}$, ou seja, independe de $t$

Notação : $y_{t} \sim \mathcal{E S T}(\mu, \gamma(h))$

\subsection{Movimento browniano e ponte browniana}

Definição 2.4 (Movimento browniano). O processo estocástico $\left\{W(t), t \in \mathbb{R}^{+}\right\}$ é um movimento browniano, ou processo de Wiener, se

1. $W(0)=0$

2. As va.s.s $\left(W\left(t_{i}\right)-W\left(t_{i-1}\right)\right)_{i=2, \ldots, k}$ são independentes para $0 \leq t_{1} \leq t_{2} \leq \ldots \leq t_{k}$

3. $(W(t)-W(s)) \sim \mathcal{N}(0, t-s)$ para $0 \leq s \leq t$

4. As trajetórias de $W(t)$ são contínuas com probabilidade 1.

A prova da existência do movimento browniano pode ser vista, por exemplo, em Billinsgsley (1995). Como consequência da definição acima, tem-se que:

$$
\operatorname{Cov}(W(s), W(t))=\min (s, t)=s \wedge t
$$

Definição 2.5 (Ponte browniana). Um processo de ponte browniana $\{B(r), 0 \leq$ $r \leq 1\}$ É um processo normal com funções amostrais contínuas $e$ : 
1. $B(0)=B(1)=0$

2. $\mathrm{E} B(r)=0$, para $0 \leq r \leq 1$

3. $\operatorname{Cov}(B(r), B(s))=r \wedge s-r s$, para $0 \leq r, s \leq 1$

A ponte browniana $B(r)$ pode ser escrita em termos do movimento browniano $W(t)$ :

$$
B(r)=W(r)-r W(1) \quad, \quad 0 \leq r \leq 1
$$

A ponte browniana de segundo nível $B_{2}(r)$ é uma generalização de $B(r)$, e em termos de $W(t)$ é definida como:

$$
B_{2}(r)=W(r)-r W(1)+6 r(1-r)\left[\frac{1}{2} W(1)-\int_{0}^{1} W(s) d s\right] \quad, \quad 0 \leq r \leq 1
$$

\subsection{Inversão numérica da função característica}

Os valores críticos e os níveis descritivos ( $p$-valores) das distribuições utilizadas neste trabalho serão obtidos pela inversão numérica de suas funções características, apresentadas na seção seguinte.

Definição 2.6 (Função característica). A função característica de uma variável aleatória $Y$, com função de distribuição $F$, é definida por

$$
\varphi_{Y}(t)=E\left(e^{i t Y}\right)=\int_{-\infty}^{\infty} e^{i t y} d F
$$

O valor de $F(y)=\mathbb{P}[Y \leq y]$ pode ser obtido pela fórmula de inversão de Lévy:

$$
F(y)-F(0)=\frac{1}{2 \pi} \int_{-\infty}^{\infty} \frac{1-e^{-i t y}}{i t} \varphi(t) d t
$$

Fórmula de inversão alternativa:

$$
F(y)=\frac{1}{2}+\frac{1}{2 \pi} \int_{0}^{\infty} \frac{e^{i t y} \varphi(-t)-e^{-i t y} \varphi(t)}{i t} d t
$$

Para o cálculo numérico da integral acima utilizamos o pacote QUADPACK ${ }^{1}$ implementado na linguagem $\mathrm{Ox}^{2}$.

\footnotetext{
${ }^{1} v$. Piessens et al. (1983)

${ }^{2}$ v. Doornik (1999)
} 


\subsection{Distribuições da família Cramér-von Mises}

O conceito relevante de convergência para as distribuições assintóticas apresentadas a seguir é o de convergência fraca de medidas de probabilidade associadas ou induzidas, que definimos abaixo. Intuitivamente, é uma generalização da convergência em distribuição para elementos aleatórios definidos em espaços mais gerais que o euclidiano.

Definição 2.7 (Convergência fraca de medidas de probabilidade associadas). Sejam $X_{n}, n \geq 0$, elementos aleatórios no espaço de probabilidade $(\Omega, \mathcal{F}, P)$ que recebem valores num espaço métrico separável e completo $(M, d)$. Uma sequência de medidas de probabilidade $P_{n}$ induzidas por $X_{n}$ converge fracamente para $P_{0}$, induzida por $X$, se, para toda função $f: M \rightarrow \mathbb{R}$ uniformemente contínua (em relação a d) e limitada, tem-se que $\int f d P_{n}=\mathrm{E} f\left(X_{n}\right) \rightarrow \mathrm{E} f(X)=\int f d P_{0}$ quando $n \rightarrow \infty$. Notação: $X_{n} \Rightarrow X$ ou $P_{n} \Rightarrow P_{0}$.

\section{Distribuição de Cramér-von Mises}

A distribuição de Cramér-von Mises, CvM, surge naturalmente no contexto de testes de qualidade de ajuste (goodness of fit tests). Mais especificamente, na avaliação assintótica da seguinte integral ${ }^{3}$ :

$$
C_{n}=\int_{-\infty}^{\infty} n\left[F_{n}(x)-F(x)\right]^{2} d F(x)=\int_{-\infty}^{\infty} U_{n}(F)^{2} d F=\int_{0}^{1} U_{n}(r)^{2} d r
$$

$F_{n}$ é a função de distribuição empírica de uma amostra aleatória $\left\{X_{1}, X_{2}, \ldots, X_{n}\right\}$ em que $X_{i}$ tem função de distribuição $F(x)$ contínua:

$$
F_{n}(x)=\frac{1}{n} \sum_{i=1}^{n} \mathbf{1}_{(-\infty, x]}\left(x_{i}\right), \quad-\infty<x<\infty,
$$

$U_{n}$ é o processo empírico uniforme:

$$
U_{n}(r)=\sqrt{n}\left[F_{U, n}(r)-r\right], \quad 0 \leq r \leq 1,
$$

\footnotetext{
${ }^{3}$ v., e.g., Shorack \& Wellner (1986)
} 
com $F_{U, n}(r)$ sendo a função de distribuição empírica de uma amostra aleatória com distribuição Uniforme $(0,1)$.

$U_{n}(r)$ converge fracamente para uma ponte browniana $B(r)$ :

$$
U_{n}(r)=\sqrt{n}\left[F_{U, n}(r)-r\right] \Rightarrow B(r)
$$

$E C_{n}$ converge então para a denominada distribuição de Cramér-von Mises ( $\left.\mathrm{V} v \mathrm{M}\right)$ :

$$
C_{n}=\int_{0}^{1} U_{n}(r)^{2} d r \Rightarrow \int_{0}^{1} B(r)^{2} d r \sim \mathrm{CvM}
$$

Função característica da distribuição CvM

Se $Y \sim$ CvM, então sua função característica $\varphi_{Y}(t)$ é dada por ${ }^{4}$ :

$$
\varphi_{Y}(t)=\sqrt{\frac{\sqrt{2 i t}}{\operatorname{sen} \sqrt{2 i t}}}
$$

Os valores da distribuição serão obtidos numericamente pela fórmula de inversão (2.6). Para tanto, convém escrever $\varphi_{Y}(t)$ em termos da função $D_{1}(x) \equiv \operatorname{sen}(\sqrt{x}) / \sqrt{x}$ :

$$
\varphi_{Y}(t)=\left[D_{1}(2 i t)\right]^{-\frac{1}{2}}
$$

Dessa forma, os valores da distribuição podem ser obtidos pela seguinte expressão, apresentada em MacNeill (1978):

$$
\mathbb{P}[Y \leq \xi]=1-\frac{1}{\pi} \sum_{j=1}^{\infty}(-1)^{j-1} \int_{\lambda_{1,2 j-1}^{-1}}^{\lambda_{1,2 j}^{-1}} \frac{e^{-\frac{x}{2} \xi} d x}{x \sqrt{-D_{1}(x)}}
$$

com

$$
\lambda_{1, k}=\frac{1}{\pi^{2} k^{2}}
$$

\footnotetext{
${ }^{4}$ v., e.g., Anderson \& Darling (1952) ou MacNeill (1978)
} 


\section{Distribuição de Cramér-von Mises de segundo nível}

Uma possível generalização da distribuição de Cramér-von Mises pode se feita pela utilização da generalização do processo de ponte browniana subjacente.

Assim, em termos do processo de ponte browniana de segundo nível $B_{2}(r)$, dado por (2.4), a distribuição de Cramér-von Mises de segundo nível $\left(\mathrm{CVM}_{2}\right)$ é definida por

$$
\int_{0}^{1} B_{2}(r)^{2} d r \sim \mathrm{CvM}_{2}
$$

Função característica da distribuição $\mathrm{CvM}_{2}$

Se $Y_{2} \sim \mathrm{CvM}_{2}$, então sua função característica $\varphi Y_{2}(t)$ é dada por $^{5}$ :

$$
\begin{aligned}
\varphi_{Y_{2}}(t) & =\left[D_{2}(2 i t)\right]^{-\frac{1}{2}} \\
D_{2}(x) & \equiv \frac{12}{x^{2}}(2-\sqrt{x} \sin \sqrt{x}-2 \cos \sqrt{x})
\end{aligned}
$$

Para o cálculo numérico da inversão de $\varphi_{Y_{2}}$, pode-se utilizar, como em MacNeill (1978), a seguinte expressão:

$$
\begin{aligned}
\mathbb{P}[Y \leq \xi] & =1-\frac{1}{\pi} \sum_{j=1}^{\infty}(-1)^{j-1} \int_{\lambda_{2,2 j-1}^{-1}}^{\lambda_{2,2}^{-1}} \frac{e^{-\frac{x}{2} \xi} d x}{x \sqrt{-D_{2}(x)}} \\
\lambda_{2,2 j-1} & =\frac{1}{4 \pi^{2} j^{2}} \\
\lambda_{2,2 j} & =\frac{1}{4 Z_{j}^{2}}
\end{aligned}
$$

$Z_{j}$ é a j-ésima raiz positiva da função esférica de Bessel de primeira espécie e ordem 1 :

$$
J_{1}(x)=\frac{\operatorname{sen} x}{x^{2}}-\frac{\cos x}{x}
$$

\footnotetext{
${ }^{5}$ v., e.g., MacNeill(1978)
} 


\section{Distribuição de Cramér-von Mises com $k$ graus de liberdade} Como em Anderson \& Darling (1952), a distribuição de Cramér-von Mises pode ainda ser representada pela seguinte expansão:

$$
\int_{0}^{1} B(r)^{2} d r \stackrel{d}{=} \sum_{j=1}^{\infty} \lambda_{1, j} \chi_{j}^{2}(1) \sim \mathrm{CvM}
$$

em que $\chi_{j}^{2}(1), j=1,2, \ldots$, são v.a.i.i.d.s com distribuição qui-quadrada com 1 grau de liberdade. Assim, a distribuição de Cramér-von Mises com $k$ graus de liberdade $\operatorname{CvM}(k)$ será definida por

$$
\sum_{j=1}^{\infty} \lambda_{1, j} \chi_{j}^{2}(k) \sim \operatorname{CvM}(k)
$$

De forma análoga, obtém-se a distribuição de Cramér-von Mises de segundo nível com $k$ graus de liberdade, $\mathrm{CvM}_{2}(k), k=1,2, \ldots$ :

$$
\sum_{j=1}^{\infty} \lambda_{2, j} \chi_{j}^{2}(k) \sim \mathrm{CvM}_{2}(k)
$$

Obs.: $\operatorname{CvM}(1)=\mathrm{CvM}$ e $\mathrm{CvM}_{2}(1)=\mathrm{CvM}_{2}$.

Funções características das distribuições $\mathrm{CvM}(k)$ e $\mathrm{CvM}_{2}(k)$

Se $X_{1} \sim \chi^{2}(m), X_{2} \sim \chi^{2}(n)$ e $X_{1}$ é independente de $X_{2}$, então $X_{1}+X_{2} \sim \chi^{2}(m+n)$. Portanto, de (2.20), tem-se que uma v.a. com distribuição $\operatorname{CvM}(k)$ equivale à soma de $k$ v.a.i.i.d.s com distribuição $\mathrm{CvM}(1)$ cada. A função característica da distribuição $\mathrm{CvM}(k)$ será dada então pelo produto de $k$ funções características da distribuição $\operatorname{CvM}(1)$ :

$$
\varphi(t)=\left(\frac{\sqrt{2 i t}}{\operatorname{sen} \sqrt{2 i t}}\right)^{\frac{k}{2}}
$$

Analogamente, a função característica da distribuição $\mathrm{CVM}_{2}(k)$ será:

$$
\begin{aligned}
\varphi(t) & =\left[D_{2}(2 i t)\right]^{-\frac{k}{2}} \\
D_{2}(x) & \equiv \frac{12}{x^{2}}(2-\sqrt{x} \sin \sqrt{x}-2 \cos \sqrt{x})
\end{aligned}
$$




\subsection{Teste invariante localmente ótimo - ILO}

\subsubsection{Teste localmente ótimo}

Seja $Y$ um vetor aleatório com densidade $f(y \mid \theta)$, $\theta$ é um parâmetro sobre o qual desejase inferir a partir do valor observado de $Y$. Quando não há um teste uniformemente mais poderoso para um teste de hipóteses unilateral do tipo:

$$
\begin{aligned}
& H_{0}: \theta \leq \theta_{0} \\
& H_{a}: \theta>\theta_{0}
\end{aligned}
$$

pode-se, seguindo Ferguson (1967), optar pelo teste localmente ótimo de Neyman e Pearson. Esse teste é localmente ótimo no sentido em que sua função poder possui a maior inclinação (derivada) em $\theta_{0}$, entre todos os testes com mesmo tamanho em $\theta_{0}$.

Seja $\phi(Y)$ um teste para (2.25). A função poder do teste $\phi(Y)$ é dada por

$$
\beta_{\phi}(\theta)=E_{\theta} \phi(Y)
$$

E sua derivada, supondo que se possa trocar a ordem entre derivação e integração,

$$
\beta_{\phi}^{\prime}(\theta)=\int \phi(y) \frac{\partial}{\partial \theta} f(y \mid \theta) d y
$$

Assim, o teste $\phi_{0}$ será localmente ótimo se, para todo teste $\phi$ de mesmo tamanho, ou seja, $\beta_{\phi}\left(\theta_{0}\right)=\beta_{\phi_{0}}\left(\theta_{0}\right)$, tem-se

$$
\beta_{\phi}^{\prime}\left(\theta_{0}\right) \leq \beta_{\phi_{0}}^{\prime}\left(\theta_{0}\right)
$$

Usando o lema de Neyman-Pearson, chega-se ao teste localmente ótimo $\phi_{0}$ que rejeita $H_{0}$ se

$$
\left.\frac{\partial}{\partial \theta} \log f(y \mid \theta)\right|_{\theta_{0}}>c_{\alpha}
$$

$c_{\alpha}$ é uma constante que depende do nível de significância $\alpha=\beta_{\phi_{0}}\left(\theta_{0}\right)$. 


\subsubsection{Teste invariante}

Seja $\mathcal{Y}$ o espaço amostral do vetor aleatório $Y$. Seja $\mathcal{G}$ um grupo de transformações (fechado, com inversa e identidade) biunívocas de $\mathcal{Y}$ em $\mathcal{Y}$. Um teste, ou uma regra de decisão, $\phi(Y)$ para testar hipóteses como (2.25) é invariante sob uma transformação $g \in \mathcal{G}$ se

$$
\phi(g Y)=\phi(Y), \forall g \in \mathcal{G}
$$

Chamamos de órbita o conjunto $\{Y: Y=g X \quad g \in \mathcal{G}\}$, $\phi$ será então invariante se e somente se $\phi$ é constante em cada órbita de $\mathcal{G}$.

Um invariante maximal é uma função $M: \mathcal{Y} \rightarrow \mathbf{R}^{k}$, para algum $k \geq 1$, que assume valor constante em cada órbita de $\mathcal{G}$ e valores distintos para diferentes órbitas.

Teorema 2.1. Seja $M$ um invariante maximal em relação ao grupo de transformações $\mathcal{G}$. Então o teste $\phi$ é invariante se e somente se existe uma função $h$ tal que $\phi(Y)=h(M(Y))$ para todo $Y \in \mathcal{Y}$.

Prova:

$' \Leftarrow{ }^{\prime} \operatorname{Se} \phi(Y)=h(M(Y))$, então $\phi$ é invariante, pois

$$
\phi(g Y)=h(M(g Y))=h(M(Y))=\phi(Y)
$$

' $\Rightarrow$ ' $M\left(Y_{1}\right)=M\left(Y_{2}\right)$ implica que $Y_{1}$ e $Y_{2}$ estão na mesma órbita e, portanto, $g Y_{1}=Y_{2}$ para algum $g \in \mathcal{G}$. Se $\phi$ é invariante, então $\phi\left(Y_{2}\right)=\phi\left(g Y_{1}\right)=\phi\left(Y_{1}\right)$. Segue então que $\phi$ é função de $M$, pois $M\left(Y_{1}\right)=M\left(Y_{2}\right) \Rightarrow \phi\left(Y_{2}\right)=\phi\left(Y_{1}\right)$ e $M\left(Y_{1}\right) \neq M\left(Y_{2}\right) \Rightarrow$ $\phi\left(Y_{2}\right) \neq \phi\left(Y_{1}\right)$

Portanto, se, em vez da distribuição de $Y$, utilizarmos a distribuição do invariante maximal $M(Y)$ na obtenção de um teste $\phi(Y)$, esse teste será invariante sob o respectivo grupo de transformações. 
O teste invariante e localmente ótimo (ILO ou LBI de locally best invariant) será então o teste localmente ótimo de Neyman-Pearson (2.29), utilizando-se a f.d.p. de $M(Y)$ em vez da f.d.p. de $Y$.

\subsubsection{Teste ILO para o modelo linear normal}

Apresentamos aqui os resultados obtidos por King \& Hillier (1985) para o modelo linear normal:

$$
\begin{aligned}
& \mathrm{y}=\boldsymbol{X} \boldsymbol{\beta}+\mathrm{u} \\
& \mathrm{u} \sim \mathcal{N}_{n}\left(0, \sigma^{2} \Omega(\theta)\right)
\end{aligned}
$$

y é um vetor aleatório $n \times 1$ e $X$ é uma matriz $n \times k$ fixa de regressores. Os parâmetros do modelo são o vetor $\boldsymbol{\beta}_{k \times 1}$ e os escalares $\sigma^{2}$ e $\theta$ por meio da forma específica da matriz de covariâncias: $\sigma^{2} \Omega(\theta)$, com $\Omega(0)=I_{n}$, a matriz identidade de dimensão $n$.

O teste de hipóteses unilateral

$$
\begin{aligned}
& H_{0}: \theta=0 \\
& H_{a}: \theta>0
\end{aligned}
$$

é invariante sob o grupo de transformações

$$
g(\mathrm{y})=\gamma_{0} \mathrm{y}+\mathbf{X} \check{\gamma}
$$

com $\gamma_{0}$ sendo um escalar positivo e $\check{\gamma}$ um vetor $k \times 1$.

O vetor $\mathrm{v} \in \mathbb{R}^{n-k}$ :

$$
\mathrm{v}=\frac{\mathbf{P}_{1} \mathbf{z}}{\left\|\mathbf{P}_{1} \mathbf{z}\right\|}
$$

é um invariante maximal em relação a (2.34), z são os resíduos do ajuste de mínimos quadrados ordinário de (2.32) e $\mathbf{P}_{1}$ projeta $\mathbf{z}$ em $\mathbb{R}^{n-k}, \mathbf{P}_{1}$ é tal que $\mathbf{P}_{1} \mathbf{P}_{1}^{\prime}=\mathbf{I}_{n-k}$ e 
$\mathbf{P}_{1}^{\prime} \mathbf{P}_{1}=\mathbf{I}_{n}-\mathbf{X}\left(\mathbf{X}^{\prime} \mathbf{X}\right)^{-1} \mathbf{X}^{\prime}$

Pode-se então obter o teste ILO a partir de (2.29) e da densidade de v:

$$
\left.\frac{\partial}{\partial \theta} \log f(\mathbf{v} \mid \theta)\right|_{\theta=0}>c_{1}
$$

Assim, chega-se ao estimador correspondente $\xi$, com a seguinte região crítica:

$$
\begin{aligned}
\xi & =\frac{\mathbf{z}^{\prime} \mathbf{A}_{0} \mathbf{Z}}{\mathbf{z}^{\prime} \mathbf{z}}<c \\
\mathbf{A}_{0} & \left.\equiv \frac{\partial}{\partial \theta} \boldsymbol{\Omega}(\theta)^{-1}\right|_{\theta=0}
\end{aligned}
$$




\section{Capítulo 3}

\section{Variância de longo termo}

\subsection{A variância de longo termo $-\sigma_{L}^{2}$}

Os testes utilizados neste trabalho (cap. 4) foram obtidos primeiramente para séries com observações independentes (ruído branco). A fim de estendê-los para séries temporais com alguma estrutura de dependência, seguiremos o trabalho de Kwiatkowski et al. (1992). Nessa linha, tem-se que os testes obtidos para séries independentes continuam válidos assintoticamente para séries com certa estrutura de dependência se substituirmos a variância amostral por uma estimativa da variância de longo termo $\sigma_{L}^{2}$, definida na sequência.

Assim, este capítulo dedica-se à obtenção de uma estimativa da variância de longo termo.

Definição 3.1 (Variância de longo termo). Seja $\varepsilon=\left\{\varepsilon_{t}\right\}_{t=1, \ldots, T}$ uma série temporal estacionária $\mathcal{E S T}(0, \gamma(h))$, ou seja, $\forall t \in\{1, \ldots, T\}$,

1. $\mathrm{E} \varepsilon_{t}=0$

2. $\operatorname{Var} \varepsilon_{t}=\sigma_{\varepsilon}^{2}=\gamma(0)<\infty$

3. $\operatorname{Cov}\left(\varepsilon_{t}, \varepsilon_{t+|h|}\right)=\gamma(h)$ 
A variancia de longo termo do processo e é definida por:

$$
\sigma_{L}^{2}=\lim _{T \rightarrow \infty} \frac{1}{T} \operatorname{Var}\left(\sum_{t=1}^{T} \varepsilon_{t}\right)=\lim _{T \rightarrow \infty} T \operatorname{Var} \bar{\varepsilon}, \quad \bar{\varepsilon}=\frac{1}{T} \sum_{t=1}^{T} \varepsilon_{t}
$$

Se $\varepsilon_{t} \sim R B\left(0, \sigma^{2}\right)$, então

$$
\begin{aligned}
\sigma_{L}^{2} & =\lim _{T \rightarrow \infty} \frac{1}{T} \operatorname{Var}\left(\sum_{t=1}^{T} \varepsilon_{t}\right)=\lim _{T \rightarrow \infty} \frac{1}{T} \sum_{t=1}^{T} \operatorname{Var} \varepsilon_{t} \\
& =\lim _{T \rightarrow \infty} \frac{1}{T} \sum_{t=1}^{T} \sigma^{2}=\lim _{T \rightarrow \infty} \frac{1}{T} T \sigma^{2}=\sigma^{2}
\end{aligned}
$$

Portanto, no caso de ruído branco, a variância de longo termo de $\varepsilon$ pode ser estimada consistentemente pela variância amostral

$$
\hat{\sigma}^{2}=\frac{1}{T} \sum_{t=1}^{T} e_{t}^{2}=\frac{e^{\prime} e}{T}, \quad e_{t}=\varepsilon_{t}-\bar{\varepsilon}
$$

A relação (3.2) deixa de valer em geral quando $\varepsilon$ possui alguma estrutura de autocorrelação. Para obter uma expressão mais geral para $\sigma_{L}^{2}$ utilizaremos o lema a seguir.

Lema de Kronecker. Se uma sequência $\left\{a_{j}\right\}_{j=0,1, \ldots}$ é tal que $\sum_{j=0}^{\infty}\left|a_{j}\right|<\infty$, então

$$
\lim _{n \rightarrow \infty} \sum_{j=0}^{n} \frac{j}{n}\left|a_{j}\right|=0
$$

Prova: Dado $\varepsilon>0$, existe um $N<\infty$ tal que $\sum_{j=N+1}^{\infty}\left|a_{j}\right|<\varepsilon$. Portanto, para $n>N$,

$\sum_{j=0}^{n} \frac{j}{n}\left|a_{j}\right|=\frac{1}{n} \sum_{j=0}^{N} j\left|a_{j}\right|+\sum_{j=N+1}^{n} \frac{j}{n}\left|a_{j}\right|<\frac{1}{n} \sum_{j=0}^{N} j\left|a_{j}\right|+\sum_{j=N+1}^{n}\left|a_{j}\right|<\frac{1}{n} \sum_{j=0}^{N} j\left|a_{j}\right|+\varepsilon$

Como, para $N$ fixo, $\lim _{n \rightarrow \infty} \frac{1}{n} \sum_{j=0}^{N} j\left|a_{j}\right|=0$, então

$$
\lim _{n \rightarrow \infty} \sum_{j=0}^{n} \frac{j}{n}\left|a_{j}\right|<\varepsilon, \quad \forall \varepsilon>0
$$


Proposição 3.1. Se $\varepsilon_{t} \sim \mathcal{E S T}(0, \gamma(h))$, com $\sum_{h=-\infty}^{\infty}|\gamma(h)|<\infty$, então

$$
\sigma_{L}^{2}=\sum_{h=-\infty}^{\infty} \gamma(h)
$$

Prova:

$$
\begin{aligned}
\sigma_{L}^{2} & =\lim _{T \rightarrow \infty} \frac{1}{T}\left[\sum_{t=1}^{T} \operatorname{Var} \varepsilon_{t}+\sum_{t=1}^{T} \sum_{t \neq r=1}^{T} \operatorname{Cov}\left(\varepsilon_{t}, \varepsilon_{r}\right)\right] \\
& =\lim _{T \rightarrow \infty} \frac{1}{T}\left[\sum_{t=1}^{T} \gamma(0)+\sum_{t=1}^{T} \sum_{t \neq r=1}^{T} \gamma(t-r)\right] \\
& =\lim _{T \rightarrow \infty} \frac{1}{T} \sum_{t=1}^{T} \sum_{r=1}^{T} \gamma(t-r) \\
& =\lim _{T \rightarrow \infty} \frac{1}{T} \sum_{h=-T+1}^{T-1}(T-|h|) \gamma(h), \text { pois } \gamma(h)=\gamma(-h) \\
& =\lim _{T \rightarrow \infty} \sum_{h=-T+1}^{T-1}\left(1-\frac{|h|}{T}\right) \gamma(h)
\end{aligned}
$$

Agora,

$$
\begin{aligned}
\left|\lim _{T \rightarrow \infty} \sum_{h=-T+1}^{T-1} \frac{|h|}{T} \gamma(h)\right| & =\left|2 \lim _{T \rightarrow \infty} \sum_{h=1}^{T-1} \frac{h}{T} \gamma(h)\right| \leq \\
& \leq 2 \lim _{T \rightarrow \infty} \sum_{h=1}^{T-1} \frac{h}{T}|\gamma(h)|=0 \text {, pelo lema de Kronecker }
\end{aligned}
$$

$E$, de (3.6),

$$
\sigma_{L}^{2}=\sum_{h=-\infty}^{\infty} \gamma(h)
$$

Se $\sum_{h=-\infty}^{\infty}|\gamma(h)|<\infty$, então $\varepsilon$ possui função densidade espectral $f(\lambda)$, definida pela tranformada de Fourier da função de autocovariância $\gamma(h)$ :

$$
f(\lambda)=\frac{1}{2 \pi} \sum_{h=-\infty}^{\infty} \gamma(h) e^{-i h \lambda}
$$


e, de (3.5)

$$
\sigma_{L}^{2}=2 \pi f(0)
$$

Sob a hipótese de que $\varepsilon$ é estacionária com densidade espectral $f(\lambda)$, tem-se que $\sigma_{L}^{2}$ pode ser estimada consistentemente por um estimador consistente de $2 \pi f(0)$. Será utilizado então um estimador suavizado de covariâncias para $f(\lambda)$ :

$$
\hat{f}(\lambda)=\frac{1}{2 \pi} \sum_{|h|<T} w(h ; l) \hat{\gamma}(h) e^{-i \lambda h}
$$

- o núcleo $w(h ; l)$ é uma função par em relação a $h$, com $|w(h ; l)| \leq w(0, l)=1$.

- l é um parâmetro positivo que indica a largura de faixa do núcleo $w(h ; l)$.

- $\hat{\gamma}(h)$ é o estimador consistente usual de $\gamma(h)$, a covariância amostral:

$$
\hat{\gamma}(h)=\frac{1}{T} \sum_{t=|h|}^{T-1} e_{t} e_{t-|h|}
$$

e $\hat{\sigma}_{L}^{2}=2 \pi \hat{f}(0)$ será o estimador para $\sigma_{L}^{2}=2 \pi f(0)$ :

$$
\hat{\sigma}_{L}^{2}=\sum_{|h|<T} w(h ; l) \hat{\gamma}(h)
$$

A consistência de $\hat{\sigma}_{L}^{2}$ será assegurada se $l \rightarrow \infty$ quando $T \rightarrow \infty$, mas com $l=o(T)$.

As escolhas do núcleo $w(h ; l)$ e da largura de faixa $l$ podem ser feitas de inúmeras formas seguindo-se apenas as restrições básicas acima. Pode-se chegar a escolhas ótimas, em algum sentido, com a imposição de outras propriedades desejáveis. Considerando núcleos da forma em que $l$ é um parâmetro de escala, ou seja, $w(h ; l)=w(h / l)$, chegase, conforme Andrews (1991), ao núcleo espectral quadrático $w_{Q S}(h / l)$ e à respectiva largura ótima $l^{*}$ minimizando-se uma expressão assintótica envolvendo o erro quadrático médio

$$
\operatorname{EQM}\left(\hat{\sigma}_{L}^{2}\right)=\mathrm{E}\left(\hat{\sigma}_{L}^{2}-\sigma_{L}^{2}\right)^{2}=\left[b\left(\hat{\sigma}_{L}^{2}\right)\right]^{2}+\operatorname{Var} \hat{\sigma}_{L}^{2}
$$


Seguindo Parzen (1957), tem-se que o viés b(.) de um estimador suavizado, como (3.11), depende da suavidade da função peso $w(x)$ em $x=0$ e da suavidade da densidade espectral $f(\lambda)$ em $\lambda=0$. Assim, definimos abaixo os números $k_{q}$ e $f_{q}$, relacionados a essas suavidades, que serão utilizados nos resultados a seguir.

A suavidade da função peso $w(x)$ em $x=0$ pode ser avaliada pelo valor de $q$ na função

$$
k_{q}=\lim _{x \rightarrow 0} \frac{1-w(x)}{|x|^{q}}, \quad q=0,1, \ldots
$$

O maior $q$ para o qual $k_{q}<\infty$, denominado expoente característico da função peso $w(x)$, é o valor de $q$ que minimiza o EQM assintótico. Isso porque, conforme argumentado em Priestley (1981), a expressão que minimiza o EQM assintótico é $O\left(T^{-\frac{2 q}{2 q+1}}\right)$, ver a proposição 3.2 abaixo. Tem-se também que quanto maior o valor do expoente característico, mais suave é a função peso na origem.

A suavidade da densidade espectral $f(\lambda)$ em $\lambda=0$ pode ser indexada por

$$
f_{q}=\frac{1}{2 \pi} \sum_{h=-\infty}^{\infty}|h|^{q} \gamma(h), \quad q=0,1, \ldots
$$

$\left|f_{q}\right|<\infty$ se e somente se $f(\lambda)$ é diferenciável $q$ vezes na frequência $\lambda=0$.

Utilizaremos ainda as seguintes classes de funções peso:

$$
\begin{aligned}
\mathcal{K}_{1}= & \{w(.): \mathbb{R} \rightarrow[-1,1] \mid w(0)=1, w(x)=w(-x) \forall x \in \mathbb{R}, \\
& \int_{-\infty}^{\infty} w^{2}(x) d x<\infty, w(.) \text { é contínua em zero e descontínua, } \\
& \text { no máximo, num número finito de pontos }\} \\
\mathcal{K}_{2}= & \left\{w(.) \in \mathcal{K}_{1} \mid W(\lambda) \geq 0 \forall \lambda \in \mathbb{R}\right\}, \quad W(\lambda) \equiv \frac{1}{2 \pi} \int_{-\infty}^{\infty} w(x) e^{-i x \lambda} d x
\end{aligned}
$$

Condição 3.1. $\left\{\varepsilon_{t}\right\}$ é uma série estacionária de quarta ordem, com média zero, $\sum_{h=-\infty}^{\infty} \gamma(h)<\infty$ e $\sum_{j=-\infty}^{\infty} \sum_{m=-\infty}^{\infty} \sum_{n=-\infty}^{\infty} \kappa(0, j, m, n)<\infty$. Em que $\kappa(t, t+$ $j, t+m, t+n)$ é o cumulante de quarta ordem de $\left(\varepsilon_{t}, \varepsilon_{t+j}, \varepsilon_{t+m}, \varepsilon_{t+n}\right)$. 
Proposição 3.2 (Parzen (1957)). Sob a condição 3.1, com $w(.) \in \mathcal{K}_{1}, l \rightarrow \infty e$ $l=o(T)$, tem-se

(i) $\lim _{T \rightarrow \infty} \frac{T}{l} \operatorname{Var} \hat{\sigma}_{L}^{2}=2\left(\sigma_{L}^{2}\right)^{2} \int_{-\infty}^{\infty} w^{2}(x) d x$

(ii) Se $\frac{l^{q}}{T} \rightarrow 0$ para algum $q<\infty$, tal que $k_{q}, f_{q}<\infty$, entâo

$$
\lim _{T \rightarrow \infty} l^{q}\left(\mathrm{E} \hat{\sigma}_{L}^{2}-\sigma_{L}^{2}\right)=-2 \pi k_{q} f_{q}
$$

(iii) Se $\frac{l^{2 q+1}}{T} \rightarrow \alpha \in(0, \infty)$ para algum $q<\infty$, tal que $k_{q}, f_{q}<\infty$, então

$$
\lim _{T \rightarrow \infty} \frac{T}{l} \operatorname{EQM}\left(\hat{\sigma}_{L}^{2}\right)=\frac{4 \pi^{2} k_{q}^{2} f_{q}^{2}}{\alpha}+2\left(\sigma_{L}^{2}\right)^{2} \int_{-\infty}^{\infty} w^{2}(x) d x
$$

Minimizando (3.15) em relação a $l$, obtém-se uma expressão para a largura ótima $l^{*}$ :

$$
l^{*}=\left[\frac{4 \pi^{2} q k_{q}^{2} f_{q}^{2} T}{\left(\sigma_{L}^{2}\right)^{2} \int_{-\infty}^{\infty} w^{2}(x) d x}\right]^{\frac{1}{2 q+1}}
$$

De Epanechnikov (1969), ou Priestley (1981), tem-se que o núcleo em $\mathcal{K}_{2}$ que minimiza (3.15) é o núcleo espectral quadrático que, seguindo a normalização de Andrews(1991) é dado por

$$
w_{Q S}(h / l)=\frac{25 l^{2}}{12 \pi^{2} h^{2}}\left[\frac{\operatorname{sen}(6 \pi h / 5 l)}{6 \pi h / 5 l}-\cos (6 \pi h / 5 l)\right]
$$

$q=2$ para o núcleo $w_{Q S}$, pois esse é o maior $q$ tal que $k_{q}<\infty$. Assim,

$$
k_{2}=\lim _{x \rightarrow 0} \frac{1-w_{Q S}(x)}{x^{2}}=-\left.\frac{1}{2 !} \frac{d^{2}}{d x^{2}} w_{Q S}(x)\right|_{x=0}=\frac{18 \pi^{2}}{125}
$$

$w_{Q S}$ dado por (3.17) já se encontra normalizado, de forma que $\int_{-\infty}^{\infty} w^{2}(x) d x=1$. Portanto, de (3.16) obtém-se a largura ótima para $w_{Q S}$ :

$$
l^{*}=\left[\frac{8 \pi^{2} k_{2}^{2} f_{2}^{2} T}{\left(\sigma_{L}^{2}\right)^{2}}\right]^{\frac{1}{5}}
$$




\subsection{Metodologia proposta para a estimação de $\sigma_{L}^{2}$}

Para a estimação de $\sigma_{L}^{2}=2 \pi f(0)$ utilizaremos o método plug-in que, conforme Andrews (1991) argumenta, é o método mais adequado, até então proposto, para uma seleção automática da largura de faixa $l$ baseada nos dados, para a estimação em uma frequência pontual, no caso em $\lambda=0$.

O método plug-in consiste em obter um estimador $\widehat{l}^{*}$ para $l^{*}$, eq. (3.19), pela substituição das quantidades desconhecidas $\sigma_{L}^{2}$ e $f_{2}=-f^{\prime \prime}(0)$ por seus respectivos estimadores:

$$
\widehat{l^{*}}\left(\hat{\sigma}_{L}^{2}, \widehat{f}_{2}\right)=\left[\frac{8 \pi^{2} k_{2}^{2} \widehat{f}_{2}^{2} T}{\left(\hat{\sigma}_{L}^{2}\right)^{2}}\right]^{\frac{1}{5}}
$$

Substituindo (3.20) em (3.11), obtém-se

$$
\hat{\sigma}_{L}^{2}=\sum_{|h|<T} w_{Q S}\left(h / \widehat{l}^{*}\left(\hat{\sigma}_{L}^{2}, \widehat{f}_{2}\right)\right) \frac{1}{T} \sum_{t=|h|}^{T-1} e_{t} e_{t-|h|}
$$

Nossa proposta é então obter o estimador $\hat{\sigma}_{L}^{2}$ resolvendo numericamente a equação acima.

Resta o problema de se encontrar um estimador para $f_{2}$. Algumas sugestões são:

1. Ajustar um modelo $\operatorname{ARMA}(\mathrm{p}, \mathrm{q})$ para $\left\{e_{t}\right\}: \Phi(L) e_{t}=\Theta(L) a_{t}, a_{t} \sim \mathrm{RB}\left(0, \sigma_{a}^{2}\right) \mathrm{e}$ utilizar os parâmetros estimados para obter uma estimativa de $f^{\prime \prime}(0)$, utilizando a relação

$$
f(\lambda)=\frac{\sigma_{a}^{2}}{2 \pi} \frac{\left|\Theta\left(e^{-i \lambda}\right)\right|^{2}}{\left|\Phi\left(e^{-i \lambda}\right)\right|^{2}}
$$

2. Ajustar localmente um polinômio em torno de $\lambda=0$ e utilizar o dobro do coeficiente quadrático como estimador de $f^{\prime \prime}(0)$. 
3. Utilizar novamente o método plug-in na relação (3.14), substituindo $\gamma(h)$ por $\hat{\gamma}(h)$ :

$$
\widehat{f_{2}}=\frac{1}{2 \pi} \sum_{|h|<T} h^{2} \hat{\gamma}(h)
$$

A sugestão 1, apresentada por Andrews (1991), não se mostrou muito adequada, pois as estimativas obtidas apresentam grandes variações para pequenas alterações dos parâmetros estimados do modelo $\operatorname{ARMA}(p, q)$.

Na sugestão 2, um bom ajuste, e uma consequente boa estimativa de $f_{2}$, ainda é muito dependente dos dados, pois o grau do melhor polinômio ajustado depende da forma de $\hat{f}(\lambda)$ em torno de $\lambda=0$.

A sugestão 3 é apresentada em Newey \& West (1994) para a estimativa de $f_{2}$, mas também para uma primeira estimativa de $\sigma_{L}^{2}$ num procedimento de duas etapas. Aqui utilizamos um procedimento distinto que apresenta estimativas finais razoavelmente estáveis.

Propomos uma variação da sugestão 3 , utilizando novamente um estimador suavizado de covariâncias com o núcleo $w_{Q S}(x)$ :

$$
\widehat{f_{2}}=\frac{1}{2 \pi} \sum_{|h|<T} h^{2} w_{Q S}\left(h / l_{2}\right) \hat{\gamma}(h)
$$

O problema agora volta a ser o original de se obter uma largura de faixa adequada, que denotaremos por $l_{2}$. Nesse estágio a estimação não é tão crítica, pois o resultado final é pouco afetado pela escolha do núcleo ou por pequenas variações da largura $l_{2}$, conforme argumentado, por exemplo, em Newey \& West (1994) ou em Sheather \& Jones (1991) no contexto de estimação de funções de densidade. Nesse sentido, utilizaremos um procedimento iterativo para obter, de forma aproximada, a menor estimativa $\widehat{l_{2}}$ para a qual $\widehat{l_{2}} \cong \widehat{l}$. 
3.3 Algoritmo para a estimação de $\sigma_{L}^{2}$

Uma idéia que surge é obter o $\widehat{l_{2}}$ que iguala $\widehat{l^{*}}$ resolvendo numericamente a equação (3.21) com $\widehat{f}_{2}$ dado por (3.24). No entanto, essa opção não se mostra viável, pois a solução numérica em geral não é única e por vezes não converge. Provavelmente, devido à alta volatilidade introduzida pelo termo $h^{2}$ em (3.24). Vale ressaltar que, nos dados reais analisados neste trabalho e nas simulações realizadas, o cálculo numérico de (3.21) sempre apresentou solução única e rápida convergência.

\subsection{Algoritmo para a estimação de $\sigma_{L}^{2}$}

O algoritmo proposto e utilizado para a estimação de $\sigma_{L}^{2}$ é então o seguinte:

1. Inicia-se com $\widehat{l_{2}}=0$.

2. Calcula-se $\widehat{f}_{2}$ pela equação (3.24), com o núcleo $w_{Q S}$ :

$$
\widehat{f_{2}}=\frac{1}{2 \pi} \sum_{|h|<T} h^{2} w_{Q S}\left(h / \widehat{l_{2}}\right) \hat{\gamma}(h)
$$

3. Calcula-se $\hat{\sigma}_{L}^{2}$, equação (3.21), numericamente pelo algoritmo de Newton-Raphson:

$$
\begin{aligned}
g\left(\hat{\sigma}_{L}^{2}\right) & \equiv \hat{\sigma}_{L}^{2}-\sum_{|h|<T} w_{Q S}\left(h ; \hat{l}^{*}\left(\hat{\sigma}_{L}^{2}, \hat{l}_{2}\right)\right) \frac{1}{T} \sum_{t=|h|}^{T-1} e_{t} e_{t-|h|} \\
\left(\hat{\sigma}_{L}^{2}\right)_{0} & =\frac{1}{T} \sum_{t=1}^{T} \epsilon_{t}^{2} \\
\left(\hat{\sigma}_{L}^{2}\right)_{i+1} & =\left(\hat{\sigma}_{L}^{2}\right)_{i}-\frac{g\left(\left(\hat{\sigma}_{L}^{2}\right)_{i}\right)}{g^{\prime}\left(\left(\hat{\sigma}_{L}^{2}\right)_{i}\right)}
\end{aligned}
$$

4. Estima-se $l^{*}$ pelo seu estimador plug-in $\widehat{l^{*}}$, dado por (3.20):

$$
\widehat{l^{*}}=\left[\frac{8 \pi^{2} k_{2}^{2} \widehat{f}_{2}^{2} T}{\left(\hat{\sigma}_{L}^{2}\right)^{2}}\right]^{\frac{1}{5}}
$$




\subsection{Algoritmo para a estimação de $\sigma_{L}^{2}$}

5. Incrementa-se $\widehat{l_{2}}$ e repete-se o procedimento a partir do item 2 até que $\widehat{l_{2}} \cong \widehat{l^{*}}$. 0 critério adotado, com bons resultados, foi $\left|\widehat{l^{*}}-\widehat{l_{2}}\right|<0.1$ 


\section{Capítulo 4}

\section{Testes de estacionariedade}

Considere o modelo de componentes não-observados:

$$
y_{t}=y_{t}^{n}+y_{t}^{a}, \quad t=1, \ldots, T
$$

em que $\mathbf{y}=\left\{y_{t}\right\}$ é a série temporal de tamanho $T$ a ser analisada, $\mathrm{y}^{n}=\left\{y_{t}^{n}\right\}$ é um componente não-aleatório e $\mathrm{y}^{a}=\left\{y_{t}^{a}\right\}$ é um componente aleatório (estocástico) a serem modelados.

Se $y^{n}=\mu_{0}+\beta t$ é uma tendência linear e $y^{a}=\mu_{t}+\varepsilon_{t}$ é a soma de um passeio aleatório $\left\{\mu_{t}\right\}$ com um processo estacionário $\left\{\varepsilon_{t}\right\}$, tem-se, na forma estrutural, o modelo com tendência linear $(M T L)$ :

$$
\begin{array}{ll}
y_{t}=\mu_{t}+\beta t+\varepsilon_{t} & , \varepsilon_{t} \sim \mathcal{E S T}\left(0, \gamma_{\varepsilon}\right) \\
\mu_{t}=\mu_{t-1}+\eta_{t} & , \quad \eta_{t} \sim \operatorname{RB}\left(0, \sigma_{\eta}^{2}\right) \\
\varepsilon_{t} \text { e } \eta_{s} \text { independentes } & , \forall t, s=1, \ldots, T
\end{array}
$$

Para testar se o componente aleatório $\mathrm{y}^{a}$ do modelo acima é estacionário, pode-se utilizar o seguinte teste de hipóteses:

$$
\begin{array}{ll}
H_{0}: & \sigma_{\eta}^{2}=0 \\
H_{1}: & \sigma_{\eta}^{2}>0
\end{array}
$$


Assim, sob a hipótese nula $H_{0}, \mu_{t}$ permanece constante e igual a $\mu_{0}$ e $\mathrm{y}^{a}$ reduz-se ao processo estacionário $\left\{\varepsilon_{t}\right\}$. Sob a hipótese alternativa, $\mu_{t}$ segue um passeio aleatório e, portanto, $\mathrm{y}^{a}$ passa a ter um componente não-estacionário, no caso uma raiz unitária.

\subsection{Série com nível constante}

Consideremos o modelo (4.2) sem tendência $(\beta=0)$ e $\varepsilon_{t}$ e $\eta_{t}$ como sendo ruídos brancos gaussianos, ou seja,

$$
\begin{array}{lll}
y_{t}=\mu_{t}+\varepsilon_{t} & , & \varepsilon_{t} \sim \mathcal{N i d}\left(0, \sigma_{s}^{2}\right) \\
\mu_{t}=\mu_{t-1}+\eta_{t} & , & \eta_{t} \sim \mathcal{N i d}\left(0, \sigma_{\eta}^{2}\right) \\
\varepsilon_{t} \quad \mathrm{e} \eta_{s} \text { independentes } & , \quad \forall t, s=1, \ldots, T
\end{array}
$$

Comparando (4.4) ao modelo linear normal (2.32) tem-se, $\operatorname{com} \theta=\frac{\sigma_{\eta}^{2}}{\sigma_{\varepsilon}^{2}}$,

$$
\Omega(\theta)=\sigma_{\varepsilon}^{2}\left[\begin{array}{lllll}
1+\theta & \theta & \theta & \ldots & \theta \\
\theta & 1+2 \theta & 2 \theta & \ldots & 2 \theta \\
\theta & 2 \theta & 1+3 \theta & \ldots & 3 \theta \\
\vdots & \vdots & \vdots & \ddots & \vdots \\
\theta & 2 \theta & 3 \theta & \cdots & 1+T \theta
\end{array}\right]
$$

e

$$
\mathbf{A}_{0}=\left.\frac{\partial \boldsymbol{\Omega}(\theta)^{-1}}{\partial \theta}\right|_{\theta=0}=-\sigma_{\varepsilon}^{2}\left[\begin{array}{ccccc}
1 & 1 & 1 & \cdots & 1 \\
1 & 2 & 2 & \cdots & 2 \\
1 & 2 & 3 & \cdots & 3 \\
\vdots & \vdots & \vdots & \ddots & \vdots \\
1 & 2 & 3 & \cdots & T
\end{array}\right]
$$

Portanto, de (2.37), obtém-se o teste invariante localmente ótimo ILO para o modelo com nivel constante (4.4):

$$
\xi_{\mu}=\frac{\mathbf{e}^{\prime} \mathbf{A}_{0} \mathbf{e}}{\mathbf{e}^{\prime} \mathbf{e}}=\frac{\sum_{i=1}^{T}\left(\sum_{t=1}^{i} e_{t}\right)^{2}}{T^{2} \hat{\sigma}_{s}{ }^{2}}>c_{\alpha}
$$


$c_{\alpha}$ é uma constante que depende do nível de significância $\alpha$ e $e_{t}$ são os resíduos do ajuste de mínimos quadrados de $y_{t}$ a um nivel constante:

$$
e_{t}=y_{t}-\bar{y}, \quad t=1, \ldots, T
$$

Isso porque, sob $H_{0},(4.4)$ se reduz a $y_{t}=\mu_{0}+\varepsilon_{t}$.

Pela aplicação do teorema de Donsker (ver, por exemplo, Shorack \& Wellner (1986)) tem-se que a soma parcial dos resíduos $e_{t}$, devidamente normalizada, converge fracamente a uma ponte browniana $B(r)$,

$$
\frac{1}{\sigma_{\varepsilon} \sqrt{T}} \sum_{i=1}^{[T r]} e_{i} \Rightarrow B(r)=W(r)-r W(1), \quad r \in[0,1]
$$

e, assintoticamente,

$$
\xi_{\mu}=\sum_{i=1}^{T}\left(\frac{\sum_{t=1}^{i} e_{t}}{T \hat{\sigma}_{\varepsilon}}\right)^{2} \Rightarrow \int_{0}^{1} B(r)^{2} d r
$$

que corresponde à distribuição de Cramér-von Mises.

\subsection{Série com tendência linear}

O acréscimo de uma tendência não-aleatória ao modelo (4.4) não altera a matriz $\Omega(\theta)$ em (4.5) e, consequentemente, o teste ILO continua a ser o dado por (4.7), porém a sua distribuição assintótica é alterada.

Considerando então o modelo com tendência linear (4.2), com $\varepsilon_{t}$ e $\eta_{t}$ sendo ruídos brancos gaussianos,

$$
\begin{array}{ll}
y_{t}=\mu_{t}+\beta t+\varepsilon_{t} & , \quad \varepsilon_{t} \sim \mathcal{N i d}\left(0, \sigma_{\varepsilon}^{2}\right) \\
\mu_{t}=\mu_{t-1}+\eta_{t} & , \quad \eta_{t} \sim \mathcal{N i d}\left(0, \sigma_{\eta}^{2}\right) \\
\varepsilon_{t} \text { e } \eta_{s} \text { independentes } & , \quad \forall t, s=1, \ldots, T
\end{array}
$$


o teste ILO será

$$
\xi_{\beta}=\frac{\mathrm{e}^{\prime} \mathbf{A}_{0} \mathrm{e}}{\mathrm{e}^{\prime} \mathrm{e}}=\frac{\sum_{i=1}^{T}\left(\sum_{t=1}^{i} e_{t}\right)^{2}}{T^{2} \hat{\sigma}_{\varepsilon}^{2}}>c_{\alpha}
$$

em que $e_{t}$ são os resíduos do ajuste de mínimos quadrados de $y_{t}$ a uma reta:

$$
\begin{aligned}
& e_{t}=y_{t}-\hat{y}_{t}, \quad t=1, \ldots, T \\
& \hat{y}_{t}=\hat{\mu}+\hat{\beta} t
\end{aligned}
$$

De MacNeill (1978), tem-se que a soma parcial desses resíduos converge para o funcional ponte browniana de segundo nível $\left(B_{2}\right)$ e, por conseguinte,

$$
\xi_{\beta} \Rightarrow \int_{0}^{1} B_{2}(r)^{2} d r=\mathrm{CvM}_{2}
$$

\subsection{Série com quebra no nível e na tendência}

Busetti \& Harvey (2001) estenderam o modelo acima de forma a permitir quebras estruturais no nível e/ou na tendência. Nessa linha, consideremos o modelo com uma quebra simultânea no nível e na tendência:

$$
\begin{array}{ll}
y_{t}=\mu_{t}+\beta t+\delta_{\mu} w_{t}+\delta_{\beta}\left(w_{t} t\right)+\varepsilon_{t} & , \varepsilon_{t} \sim \mathcal{N} \mathrm{id}\left(0, \sigma_{\varepsilon}^{2}\right) \\
\mu_{t}=\mu_{t-1}+\eta_{t} & , \quad \eta_{t} \sim \mathcal{N} \mathrm{id}\left(0, \sigma_{\eta}^{2}\right) \\
\varepsilon_{t} \mathrm{e} \eta_{s} \text { independentes } & , \forall t, s=1, \ldots, T
\end{array}
$$

em que $w_{t}=\mathbf{1}_{\{t>\tau\}}$ e $\tau$ é o instante, conhecido, em que ocorre uma mudança estrutural.

O teste ILO continua a ser dado pela mesma estatística:

$$
{ }_{1} \xi_{\beta}(\lambda)=\frac{\sum_{i=1}^{T}\left(\sum_{t=1}^{i} e_{t}\right)^{2}}{T^{2} \hat{\sigma}_{\varepsilon}^{2}}>c_{\alpha}
$$

cuja distribuição assintótica agora depende de $\lambda=\tau / T$. O índice 1 em ${ }_{1} \xi_{\beta}$ denota a presença de uma quebra. 
Pela Proposição 3.2 de Busetti \& Harvey (2001) tem-se que

$$
\begin{aligned}
{ }_{1} \xi_{\beta}(\lambda) & =\lambda^{2} \frac{\sum_{i=1}^{\tau}\left(\sum_{t=1}^{i} e_{t}\right)^{2}}{\tau^{2} \hat{\sigma}_{\varepsilon}^{2}}+(1-\lambda)^{2} \frac{\sum_{i=\tau+1}^{T}\left(\sum_{t=\tau+1}^{i} e_{t}\right)^{2}}{(T-\tau)^{2} \hat{\sigma}_{\varepsilon}^{2}} \\
{ }_{1} \xi_{\beta}(\lambda) & \Rightarrow \lambda^{2} \int_{0}^{1} B_{2}(r)^{2} d r+(1-\lambda)^{2} \int_{0}^{1} B_{2}^{\prime}(r)^{2} d r
\end{aligned}
$$

em que $B_{2}(r)$ e $B_{2}^{\prime}(r)$ são pontes brownianas de segundo nível independentes.

Portanto, a distribuição assintótica de ${ }_{1} \xi_{\beta}(\lambda)$ é uma combinação linear de duas distribuições $\mathrm{CvM}_{2}$. De (2.17), a função característica correspondente será dada por

$$
\begin{aligned}
\varphi_{1} \xi_{\beta}(t) & =\left[D_{2}\left(2 i t \lambda^{2}\right) D_{2}\left(2 i t(1-\lambda)^{2}\right)\right]^{-\frac{1}{2}} \\
D_{2}(x) & \equiv \frac{12}{x^{2}}(2-\sqrt{x} \operatorname{sen} \sqrt{x}-2 \cos \sqrt{x})
\end{aligned}
$$

\section{Teste simplificado para mais de uma quebra}

O procedimento acima para o cálculo da região crítica torna-se bastante trabalhoso se pretende-se modelar uma série com mais de uma quebra. Busetti \& Harvey (2001) propõem então um teste simplificado: eles constataram que a estatística (4.18) sem o parâmetro $\lambda$

$$
{ }_{1} \xi_{\beta}^{*}=\frac{\sum_{i=1}^{\tau}\left(\sum_{t=1}^{i} e_{t}\right)^{2}}{\tau^{2} \hat{\sigma}_{\varepsilon}^{2}}+\frac{\sum_{i=\tau+1}^{T}\left(\sum_{t=\tau+1}^{i} e_{t}\right)^{2}}{(T-\tau)^{2} \hat{\sigma}_{\varepsilon}^{2}}
$$

apresenta tamanho e poder semelhantes para $0.1 \leq \lambda \leq 0.9$. Além disso, ${ }_{1} \xi_{\beta}^{*}$ sugere uma generalização para o modelo com $k$ quebras no nível e na tendência:

$$
{ }_{k} \xi_{\beta}^{*}=\sum_{j=1}^{k+1} \frac{\sum_{i=\tau_{j-1}+1}^{\tau_{j}}\left(\sum_{t=\tau_{j-1}+1}^{i} e_{t}\right)^{2}}{\left(\tau_{j}-\tau_{j-1}\right)^{2} \hat{\sigma}_{\varepsilon}^{2}}
$$

em que $0=\tau_{0}<\tau_{1}<\ldots<\tau_{k}<T$ indicam os instantes em que se permite uma quebra simultânea no nível e na tendência linear. 
Então, a distribuição assintótica de ${ }_{k} \xi_{\beta}^{*}$ será a mesma que a da soma de $k+1$ variáveis aleatórias independentes com distribuição $\mathrm{CvM}_{2}$ cada. Essa distribuição é denotada por $\mathrm{CvM}_{2}(k+1)$ em Busetti \& Harvey (2001). E sua função característica será então

$$
\begin{aligned}
\varphi_{k} \xi_{\beta}^{*}(t) & =\left[D_{2}(2 i t)\right]^{-\frac{k+1}{2}} \\
D_{2}(x) & \equiv \frac{12}{x^{2}}(2-\sqrt{x} \sin \sqrt{x}-2 \cos \sqrt{x})
\end{aligned}
$$

\subsection{Série com quebra no nível apenas}

Também de Busetti \& Harvey (2001), tem-se o modelo mais simples, com quebra no nível apenas:

$$
\begin{array}{ll}
y_{t}=\mu_{t}+\delta_{\mu} w_{t}+\varepsilon_{t} & , \quad \varepsilon_{t} \sim \mathcal{N i d}\left(0, \sigma_{\varepsilon}^{2}\right) \\
\mu_{t}=\mu_{t-1}+\eta_{t} & , \quad \eta_{t} \sim \mathcal{N i d}\left(0, \sigma_{\eta}^{2}\right) \\
\varepsilon_{t} \text { e } \eta_{s} \text { independentes } & , \forall t, s=1, \ldots, T
\end{array}
$$

com $w_{t}=\mathbf{1}_{\{t>\tau\}}$ e $\tau$ é o instante, conhecido, em que ocorre a quebra no nível.

O teste ILO continua a ser dado pela mesma estatística:

$$
{ }_{1} \xi_{\mu}(\lambda)=\frac{\sum_{i=1}^{T}\left(\sum_{t=1}^{i} e_{t}\right)^{2}}{T^{2} \hat{\sigma}_{\varepsilon}^{2}}>c_{\alpha}
$$

e sua distribuição assintótica será a combinação linear de duas v.a.s independentes com distribuição CvM cada. De (2.13), a função característica correspondente será dada por

$$
\begin{aligned}
\varphi_{1 \xi_{\mu}}(t) & =\left[D_{1}\left(2 i t \lambda^{2}\right) D_{1}\left(2 i t(1-\lambda)^{2}\right)\right]^{-\frac{1}{2}} \\
D_{1}(x) & \equiv \frac{\operatorname{sen} \sqrt{x}}{\sqrt{x}}
\end{aligned}
$$




\section{Teste simplificado para mais de uma quebra}

Analogamente, o teste simplificado para $k$ quebras no nível no modelo (4.24) será

$$
{ }_{k} \xi_{\mu}^{*}=\sum_{j=1}^{k+1} \frac{\sum_{i=\tau_{j-1}+1}^{\tau_{j}}\left(\sum_{t=\tau_{j-1}+1}^{i} e_{t}\right)^{2}}{\left(\tau_{j}-\tau_{j-1}\right)^{2} \hat{\sigma}_{\varepsilon}^{2}}
$$

e a distribuição assintótica de ${ }_{k} \xi_{\mu}^{*}$ tem a função característica

$$
\varphi_{k} \xi_{\mu}^{*}(t)=\left[D_{1}(2 i t)\right]^{-\frac{k+1}{2}}
$$

\subsection{Extensão para ruídos estacionários}

Condição 4.1 (Phillips \& Perron (1988)). Seja $\left\{\varepsilon_{t}\right\}_{t=1, \ldots, \infty}$ uma série temporal satisfazendo

1. $\mathrm{E} \varepsilon_{t}=0, \forall t$

2. $\sup _{t} \mathrm{E}\left|\varepsilon_{t}\right|^{\beta}<\infty$, para algum $\beta>2$;

3. $\sigma_{L}^{2}=\lim _{T \rightarrow \infty} T^{-1} \operatorname{Var}\left(\sum_{t=1}^{T} \varepsilon_{t}\right)$ existe $e \sigma_{L}^{2}>0$;

4. $\left\{\varepsilon_{t}\right\}_{t=1, \ldots, \infty}$ é um strong mixing, com coeficientes de mixing $\alpha_{m}$ satisfazendo $\sum_{m=1}^{\infty} \alpha_{m}^{1-2 / \beta}<\infty$.

Se os processos $\left\{\varepsilon_{t}\right\}$ nos modelos apresentados anteriormente respeitam a condição 4.1, então as estatísticas $\xi s$ correspondentes apresentam as mesmas distribuições assintóticas se substituirmos $\hat{\sigma}_{\varepsilon}^{2}$ por um estimador consistente da variância de longo termo $\sigma_{L}^{2}$. Isso porque

$$
\frac{1}{\sqrt{T}} \sum_{t=1}^{[T r]} e_{t} \Rightarrow \sigma_{L}^{2} B(r)
$$

no caso em que $e_{t}=y_{t}-\bar{y}, \mathrm{e}$

$$
\frac{1}{\sqrt{T}} \sum_{t=1}^{[T r]} e_{t} \Rightarrow \sigma_{L}^{2} B_{2}(r)
$$


no caso em que $e_{t}=y_{t}-\hat{\mu}-\hat{\beta} t$.

Para mais detalhes, ver, por exemplo, Kwiatkovski et al. (1992).

\subsection{Modelos com componente sazonal}

Conforme demonstrado em Busetti (2002), a inclusão de um componente sazonal determinístico nos modelos anteriores não altera as distribuições assintóticas das respectivas estatísticas apresentadas.

O componente sazonal determinístico $\left\{z_{t}\right\}$ pode ser parametrizado, por exemplo, com a utilização de dummies sazonais:

$$
\begin{aligned}
z_{t} & =\sum_{i=1}^{s-1} \omega_{i} z_{i t} \\
z_{i t} & =\mathbf{1}_{\{t=i+n s\}}-\mathbf{1}_{\{t=n s\}}, \quad n=0,1,2, \ldots, \quad i=1,2, \ldots, s-1
\end{aligned}
$$

$s$ é o período sazonal. 


\section{Capítulo 5}

\section{Simulações}

\subsection{Estimativa da variância de longo termo}

A fim de avaliar a qualidade da estimativa da variância de longo termo $\sigma_{L}^{2}$, proposta no capítulo 3, realizamos um experimento de Monte Carlo em um processo $\operatorname{AR}(1)$, para o qual se tem a fórmula exata de $\sigma_{L}^{2}$.

Seja $\left\{\varepsilon_{t}\right\}_{t=1, \ldots, T}$ um processo $\operatorname{AR}(1)$ com parâmetro $\phi$ :

$$
\varepsilon_{t}=\phi \varepsilon_{t-1}+a_{t}, \quad a_{t} \sim \mathcal{N i d}(0,1)
$$

Utilizando as relações (3.8) e (3.22), $\sigma_{L}^{2}$ será dada por

$$
\begin{aligned}
\sigma_{L}^{2} & =2 \pi f(0) \\
& =2 \pi \frac{1}{2 \pi} \frac{1}{1-2 \phi \cos 0+\phi^{2}} \\
& =\frac{1}{(1-\phi)^{2}}, \quad|\phi|<1
\end{aligned}
$$

A tabela 5.1 apresenta os valores médios de $\hat{\sigma}_{L}^{2}$ obtidos de 1000 processos (5.1) simulados de tamanho $T$ e parâmetro $\phi$. A tabela 5.2 traz os valores médios de $\hat{l}^{*}$. 
Tabela 5.1: Estimativas médias da variância de longo termo $\sigma_{L}^{2}$ para 1000 amostras simuladas de tamanho $T$ de um processo AR(1) com parâmetro $\phi$.

\begin{tabular}{c|cccc}
\hline & \multicolumn{4}{|c}{$\phi$} \\
$\mathrm{T}$ & 0 & 0.2 & 0.5 & 0.8 \\
\hline 100 & 0.988 & 1.45 & 3.18 & 14.6 \\
200 & 0.997 & 1.48 & 3.43 & 17.2 \\
500 & 0.992 & 1.51 & 3.64 & 19.5 \\
1000 & 0.996 & 1.53 & 3.77 & 20.9 \\
\hline \hline$\sigma_{L}^{2}$ & 1 & 1.56 & 4 & 25 \\
\hline
\end{tabular}

Comparando-se os valores médios de $\hat{\sigma}_{L}^{2}$ com o valor nominal de $\sigma_{L}^{2}$ (última linha da tabela 5.1), nota-se que em média as estimativas são menores que os valores nominais e os resultados são melhores para as séries com menor persistência ( $\phi$ menor). Verifica-se também aproximações melhores para amostras maiores, o que é esperado, pois o valor nominal de $\sigma_{L}^{2}$ é um resultado assintótico.

Tabela 5.2: Estimativas médias da largura ótima $l^{*}$ para 1000 amostras simuladas de tamanho $T$ de um processo $\operatorname{AR}(1)$ com parâmetro $\phi$.

\begin{tabular}{c|cccc}
\hline & \multicolumn{4}{|c}{$\phi$} \\
$\mathrm{T}$ & 0 & 0.2 & 0.5 & 0.8 \\
\hline 100 & 4.8 & 4.7 & 4.7 & 6.5 \\
200 & 6.6 & 6.3 & 6.6 & 8.9 \\
500 & 9.6 & 9.5 & 9.4 & 13.0 \\
1000 & 13.4 & 12.8 & 13.0 & 16.4 \\
\hline
\end{tabular}

Quanto aos valores médios obtidos para $\widehat{l}^{*}$ (tabela 5.2), nota-se um aumento para as séries mais persistentes $(\phi=0.8)$, o que também é esperado, pois quanto mais persistente a série, maior deveria ser a largura de faixa dos núcleos, para se dar maior peso às informações passadas. 


\subsection{Tamanho e poder para amostras finitas}

Apresentamos aqui os resultados obtidos por experimento de Monte Carlo para avaliar empiricamente o tamanho e o poder do teste para séries com uma quebra simultânea no nível e na tendência (seção 4.3). Novamente considerando o ruído estacionário $\left\{\varepsilon_{t}\right\}$ como um processo $A R(1)$, simulamos 1000 réplicas do processo

$$
\begin{aligned}
& y_{t}=\mu_{t}+2 t-[T / 2] w_{t}-w_{t} t+\varepsilon_{t} \quad, \quad \varepsilon_{t}=\phi \varepsilon_{t-1}+a_{t}, \quad a_{t} \sim \mathcal{N} \mathrm{id}(0,1) \\
& \mu_{t}=\mu_{t-1}+\eta_{t} \quad, \eta_{t} \sim \mathcal{N i d}(0, \theta) \\
& w_{t}=\mathbf{1}_{\{t>[\lambda T]\}} \quad, t=1, \ldots, T
\end{aligned}
$$

Ou seja, fixamos os parâmetros $\mu_{0}=0, \beta=2, \delta_{\mu}=-[T / 2], \delta_{\beta}=-1$ e $\sigma_{a}^{2}=1$.

O teste ILO rejeita $H_{0}: \theta=0$ para

$$
{ }_{1} \xi_{\beta}(\lambda)=\frac{\sum_{i=1}^{T}\left(\sum_{t=1}^{i} e_{t}\right)^{2}}{T^{2} \hat{\sigma}_{L}^{2}}>c_{\alpha}
$$

$\operatorname{com} \hat{\sigma}_{L}^{2}$ obtido conforme o algoritmo da seção 3.3 .

Os resultados obtidos para um tamanho nominal $\alpha=5 \%$ encontram-se nas tabelas 5.3 e 5.4 , com $\lambda=0.5$ e $\lambda=0.1$, respectivamente.

Em linhas gerais, tem-se melhores resultados para um maior tamanho da amostra (eficiência assintótica) e para uma menor persistência do ruído ( $\phi$ menor). Esperase do poder que ele aumente monotonicamente ao distanciarmo-nos de $H_{0}: \quad \theta=0$ e tenda a 1 para $\theta \rightarrow \infty$. Essas características não se verificam aqui. Tipicamente, o poder apresenta um máximo para $\theta=0.1$ ou 1 e volta a cair tendendo para um valor diferente de 1. Na figura 5.1 apresentamos esse comportamento mais detalhadamente para $\lambda=0.1, \phi=0, T=100,10^{-4} \leq \theta \leq 10^{3}$ e 5000 réplicas. Uma explicação possível é que para valores mais altos de $\theta$, com uma maior predominância relativa do componente de passeio aleatório, há uma maior probabilidade de ocorrer trechos de tendência 
estocástica passíveis de serem espuriamente bem ajustados pelo método utilizado de mínimos quadrados ordinários. Essa explicação é corroborada se observarmos que os resultados obtidos para $\lambda=0.1$ (tabela 5.4) são melhores do que os para $\lambda=0.5$ (tabela 5.3). Isso porque, por questão de simetria, em um passeio aleatório é mais provável a existência de um bom ajuste espúrio com quebra no meio da amostra do que com quebra em uma extremidade, no caso em $10 \%$ da amostra.

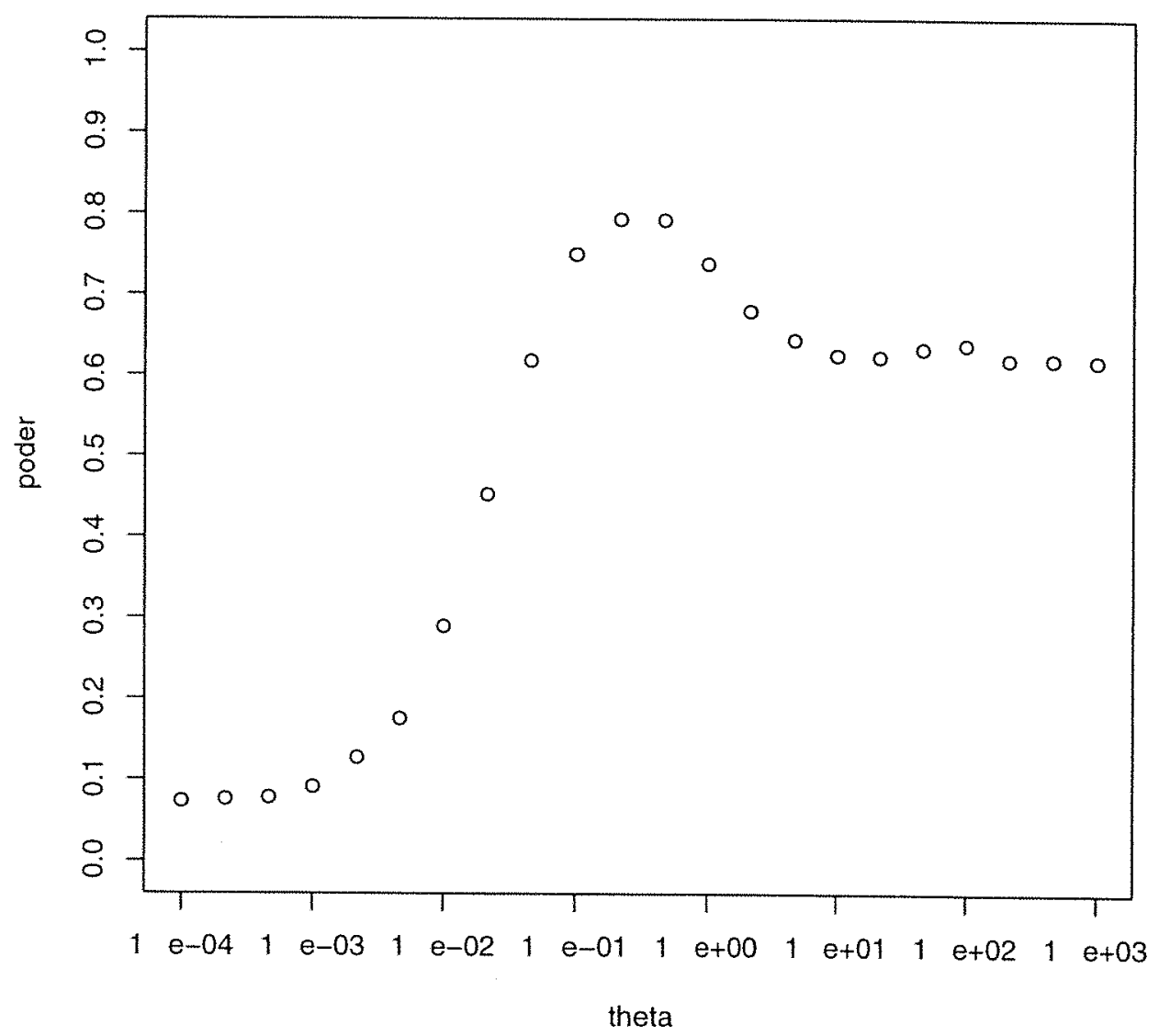

Figura 5.1: Poder empírico para o teste (5.4) com $\lambda=0.1, \phi=0, T=100,5000$ réplicas e $10^{-4} \leq \theta \leq 10^{3}$ ( $\theta$ em escala logarítmica) 
Tabela 5.3: Tamanho $(\theta=0)$ e poder $(\theta>0)$ empíricos para o teste $(5.4), \lambda=0.5$ e 1000 réplicas.

\begin{tabular}{c|c|cccc}
\hline & & \multicolumn{4}{|c}{$\phi$} \\
& $\theta$ & 0 & 0.2 & 0.5 & 0.8 \\
\hline$T=100$ & 0.1 & 0.087 & 0.137 & 0.289 & 0.419 \\
$\lambda=0.5$ & 1 & 0.714 & 0.699 & 0.668 & 0.540 \\
& 10 & 0.604 & 0.594 & 0.606 & 0.585 \\
\hline \multirow{3}{*}{$T=200$} & 0 & 0.058 & 0.111 & 0.276 & 0.366 \\
$\lambda=0.5$ & 0.1 & 0.878 & 0.846 & 0.765 & 0.512 \\
& 1 & 0.712 & 0.693 & 0.719 & 0.655 \\
& 10 & 0.643 & 0.628 & 0.667 & 0.642 \\
\hline & 0 & 0.049 & 0.084 & 0.221 & 0.315 \\
$\lambda=500$ & 0.1 & 0.854 & 0.874 & 0.919 & 0.801 \\
& 1 & 0.715 & 0.720 & 0.731 & 0.785 \\
& 10 & 0.707 & 0.701 & 0.702 & 0.720 \\
\hline
\end{tabular}

Tabela 5.4: Tamanho $(\theta=0)$ e poder $(\theta>0)$ empíricos para o teste $(5.4), \lambda=0.1$ e 1000 réplicas.

\begin{tabular}{|c|c|c|c|c|c|}
\hline & \multirow[b]{2}{*}{$\theta$} & \multicolumn{4}{|c|}{$\phi$} \\
\hline & & 0 & 0.2 & 0.5 & 0.8 \\
\hline & 0 & 0.062 & 0.122 & 0.251 & 0.374 \\
\hline$T=100$ & 0.1 & 0.745 & 0.717 & 0.613 & 0.463 \\
\hline \multirow[t]{3}{*}{$\lambda=0.1$} & 1 & 0.718 & 0.704 & 0.714 & 0.603 \\
\hline & 10 & 0.644 & 0.639 & 0.632 & 0.631 \\
\hline & 0 & 0.044 & 0.078 & 0.190 & 0.326 \\
\hline$T=200$ & 0.1 & 0.874 & 0.863 & 0.816 & 0.606 \\
\hline \multirow[t]{2}{*}{$\lambda=0.1$} & 1 & 0.710 & 0.710 & 0.718 & 0.692 \\
\hline & 10 & 0.682 & 0.683 & 0.692 & 0.682 \\
\hline
\end{tabular}




\subsection{Tamanho e poder do teste simplificado}

Além dos testes apresentados no capítulo 4, Busetti \& Harvey (2001) apresentam também os testes para quebra apenas na tendência linear ou apenas no nível no modelo com tendência. Ocorre no entanto que é mais trabalhosa a obtenção das distribuições assintóticas, via inversão da função característica, das respectivas estatísticas de teste. $E$ também não é possível obter uma estatística simplificada como a apresentada nas seções 4.3 e 4.4, isso porque não é possível separar a distribuição em duas ou mais distribuições independentes ${ }^{1}$.

A idéia então é utilizar o teste simplificado para quebra simultânea no nível e na tendência (estatística ${ }_{1} \xi_{\beta}^{*}$ ) em séries geradas com quebra apenas na tendência, ou seja, a série é 'contínua' mas apresenta uma mudança de tendência em $t=\tau$.

Para comparar o desempenho dos dois testes, simulamos séries com quebra apenas na tendência e obtivemos o tamanho e o poder empírico dos testes para 10000 réplicas e um tamanho nominal $\alpha=5 \%$.

O processo gerador de dados é:

$$
\begin{array}{ll}
y_{t}=\mu_{t}+t+z_{t}+\varepsilon_{t} & , \quad \varepsilon_{t}=\phi \varepsilon_{t-1}+a_{t}, \\
\mu_{t}=\mu_{t-1}+\eta_{t} & a_{t} \sim \mathcal{N i d}(0,1) \\
z_{t}=(t-\tau) \mathbf{1}_{\{t>\tau\}} & , \quad t=1, \ldots, T
\end{array}
$$

Estatística do teste simplificado com quebra simultânea no nível e na tendência

$$
{ }_{1} \xi_{\beta}^{*}=\frac{\sum_{i=1}^{\tau}\left(\sum_{t=1}^{i} e_{t}\right)^{2}}{\tau^{2} \hat{\sigma}_{\varepsilon}^{2}}+\frac{\sum_{i=\tau+1}^{T}\left(\sum_{t=\tau+1}^{i} e_{t}\right)^{2}}{(T-\tau)^{2} \hat{\sigma}_{\varepsilon}^{2}}
$$

\footnotetext{
${ }^{1}$ ver também Kurozumi (2002)
} 
Os resíduos $\left\{e_{t}\right\}$ são obtidos do ajuste de mínimos quadrados

$$
\begin{aligned}
& y_{t}=\hat{\mu}_{0}+\hat{\beta} t+\hat{\delta}_{\mu} w_{t}+\hat{\delta}_{\beta} w_{t} t+e_{t} \\
& w_{t}=1_{\{t>\tau\}}
\end{aligned}
$$

Estatística do teste com quebra apenas na tendência

$$
{ }_{1} \xi_{\beta}^{\prime}=\frac{\sum_{i=1}^{T}\left(\sum_{t=1}^{i} e_{t}\right)^{2}}{T^{2} \hat{\sigma}_{\varepsilon}^{2}}
$$

Os resíduos $\left\{e_{t}\right\}$ são obtidos do ajuste de mínimos quadrados

$$
\begin{aligned}
& y_{t}=\hat{\mu}_{0}+\hat{\beta} t+\hat{\delta}_{\beta} z_{t} t+e_{t} \\
& z_{t}=(t-\tau) \mathbf{1}_{\{t>\tau\}}
\end{aligned}
$$

Os valores críticos da distribuição assintótica de ${ }_{1} \xi_{\beta}^{\prime}$, para um tamanho nominal de $5 \%$, são $0.12716,0.10956,0.09563,0.08643$ e 0.08318 para $\lambda=0.1,0.2,0.3,0.4$ e 0.5 , respectivamente (Kurozumi (2002)).

Como se depreende dos resultados obtidos (tabelas 5.5, 5.6 e 5.7), o teste simplificado apresenta uma leve vantagem em termos de tamanho (linhas $\operatorname{com} \theta=0$ ). $E$ o teste "exato" apresenta uma vantagem, também não muito grande, em termos de poder (linhas $\operatorname{com} \theta>0$ ). Ambos os testes apresentam maior poder com o aumento da amostra, de $T=100$ para $T=200$, como esperado, pois a distribuição utilizada é assintótica.

Assim, pelos resultados dessas simulações, o teste simplificado para quebra no nível e na tendência aplicado a séries com quebra apenas na tendência apresenta desempenho semelhante do teste derivado para quebra apenas na tendência e que leva em conta a localização da quebra. 
Tabela 5.5: Tamanho $(\theta=0)$ e poder $(\theta>0)$ empíricos, 10000 réplicas.

\begin{tabular}{c|c|ccccc}
\hline \hline \multirow{2}{*}{$T=100$} & & \multicolumn{5}{|c}{$\lambda=\tau / T$} \\
& $\theta$ & 0.1 & 0.2 & 0.3 & 0.4 & 0.5 \\
\hline teste & 0 & 0.0532 & 0.0524 & 0.0537 & 0.0527 & 0.0520 \\
simplificado & 0.01 & 0.2365 & 0.2192 & 0.1979 & 0.1770 & 0.1709 \\
com quebra & 0.1 & 0.7840 & 0.7644 & 0.7627 & 0.7676 & 0.7608 \\
no nivel e & 1 & 0.9752 & 0.9824 & 0.9898 & 0.9906 & 0.9915 \\
na tendência & 10 & 0.9908 & 0.9968 & 0.9987 & 0.9992 & 0.9991 \\
\hline \hline teste & 0 & 0.0567 & 0.0545 & 0.0510 & 0.0564 & 0.0503 \\
com quebra & 0.01 & 0.3281 & 0.2971 & 0.2572 & 0.2388 & 0.2153 \\
na tendência & 0.1 & 0.8692 & 0.8340 & 0.8028 & 0.8060 & 0.7952 \\
apenas & 1 & 0.9919 & 0.9890 & 0.9891 & 0.9913 & 0.9911 \\
& 10 & 0.9989 & 0.9985 & 0.9979 & 0.9984 & 0.9985 \\
\hline
\end{tabular}

Tabela 5.6: Tamanho $(\theta=0)$ e poder $(\theta>0)$ empíricos, 10000 réplicas.

\begin{tabular}{c|c|ccccc}
\hline \multirow{2}{*}{$T=200$} & & \multicolumn{5}{|c}{$\lambda=\tau / T$} \\
& $\theta$ & 0.1 & 0.2 & 0.3 & 0.4 & 0.5 \\
\hline teste & 0 & 0.0515 & 0.0499 & 0.0515 & 0.0485 & 0.0515 \\
simplificado & 0.01 & 0.5980 & 0.5577 & 0.5249 & 0.5270 & 0.5084 \\
com quebra & 0.1 & 0.9708 & 0.9771 & 0.9794 & 0.9859 & 0.9840 \\
no nível e & 1 & 0.9997 & 0.9996 & 0.9999 & 1 & 0.9998 \\
na tendência & 10 & 0.9998 & 1 & 1 & 1 & 1 \\
\hline \hline teste & 0 & 0.0533 & 0.0585 & 0.0531 & 0.0520 & 0.0481 \\
com quebra & 0.01 & 0.6906 & 0.6528 & 0.6007 & 0.5878 & 0.5789 \\
na tendência & 0.1 & 0.9877 & 0.9827 & 0.9799 & 0.9855 & 0.9857 \\
apenas & 1 & 0.9998 & 0.9998 & 0.9999 & 1 & 1 \\
& 10 & 1 & 1 & 1 & 1 & 1 \\
\hline
\end{tabular}


Tabela 5.7: Tamanho $(\theta=0)$ e poder $(\theta>0)$ empíricos, 10000 réplicas.

\begin{tabular}{c|c|ccccc}
\hline \hline$T=500$ & & \multicolumn{5}{|c}{$\lambda=\tau / T$} \\
& $\theta$ & 0.1 & 0.2 & 0.3 & 0.4 & 0.5 \\
\hline teste & 0 & 0.0509 & 0.0496 & 0.0461 & 0.0493 & 0.0518 \\
simplificado & 0.01 & 0.9611 & 0.9625 & 0.9645 & 0.9652 & 0.9681 \\
com quebra & 0.1 & 1 & 1 & 1 & 1 & 1 \\
no nivel e & 1 & 1 & 1 & 1 & 1 & 1 \\
na tendência & 10 & 1 & 1 & 1 & 1 & 1 \\
\hline \hline teste & 0 & 0.0537 & 0.0491 & 0.0476 & 0.0506 & 0.0494 \\
com quebra & 0.01 & 0.9781 & 0.9720 & 0.9672 & 0.9682 & 0.9719 \\
na tendência & 0.1 & 1 & 1 & 1 & 1 & 1 \\
apenas & 1 & 1 & 1 & 1 & 1 & 1 \\
& 10 & 1 & 1 & 1 & 1 & 1 \\
\hline
\end{tabular}




\section{Capítulo 6}

\section{Aplicações}

\subsection{Análise das exportações brasileiras}

O objetivo é analisar a série mensal de exportações brasileiras nas últimas décadas a fim de testar seu comportamento, utilizando-se da metodologia apresentada. Ou seja, desejase testar se o componente estocástico da série é ou não estacionário quando se modela a parte não-aleatória com componentes sazonais e de tendência linear. Permitindo-se ainda uma ou mais quebras estruturais no nível e na tendência.

Visto que a série claramente apresenta uma tendência crescente e que a variabilidade é proporcional ao nível (fig.6.1), trabalharemos com o logaritmo da série, que possui uma variabilidade mais regular (fig.6.2).

Assim, será utilizada a série de logaritmos (na base 10) das exportações mensais brasileiras, $x_{t}=\log _{10} X_{t}$, em dólar corrente, do período de janeiro/1969 a dezembro/2003. 


\subsection{Análise das exportações brasileiras}

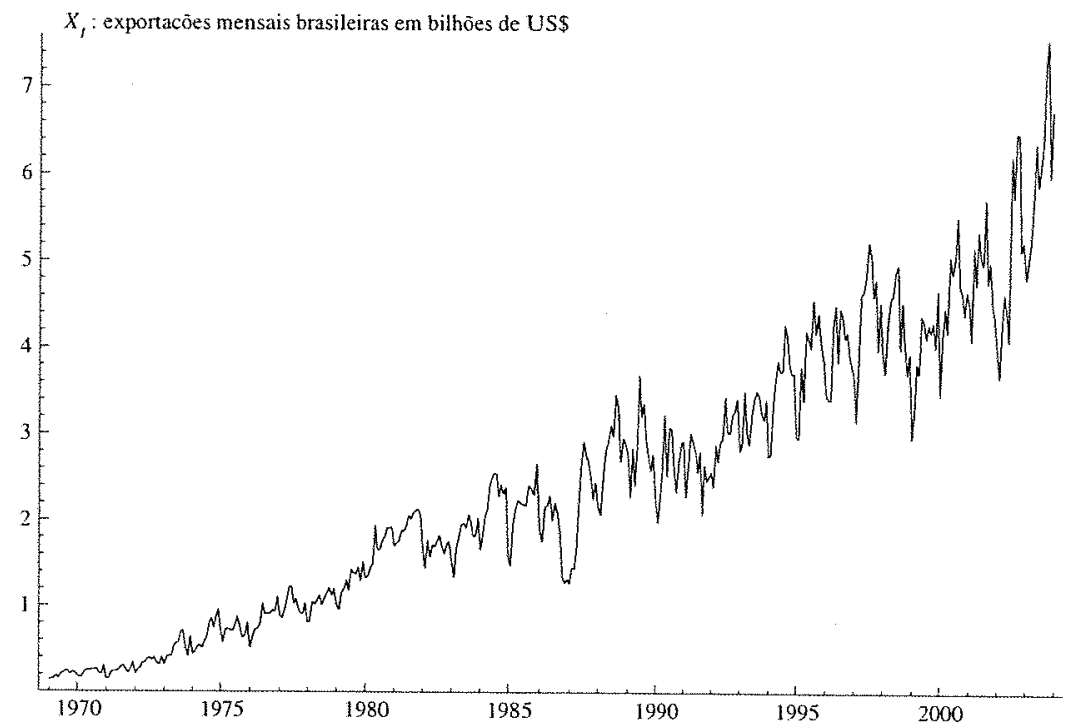

Figura 6.1: $X_{t}=$ Exportações mensais brasileiras, em bilhões de US\$, do período de janeiro/1969 a dezembro/2003, $t=1, \ldots, T=420$

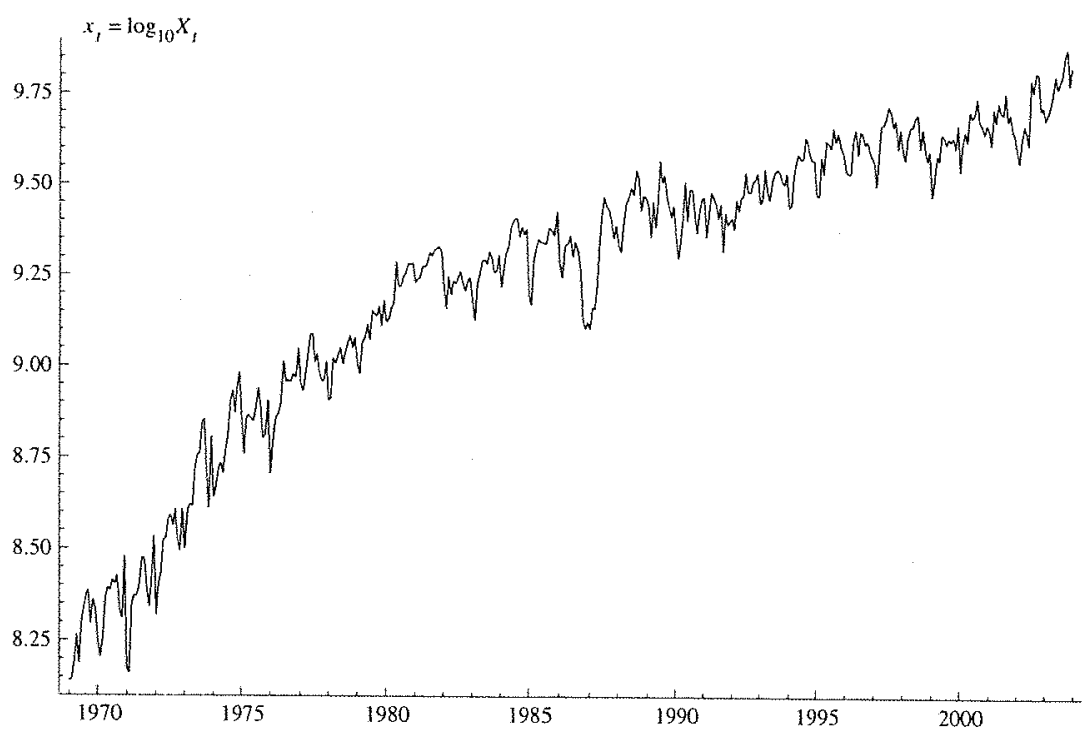

Figura 6.2: $x_{t}=\log _{10} X_{t}$ 


\section{Modelo com tendência e sem quebras estruturais}

Como primeira abordagem, testou-se o modelo com uma tendência linear, mas sem quebras estruturais:

$$
\begin{aligned}
& x_{t}=\mu_{t}+\beta t+\omega_{t}+\varepsilon_{t} \quad, \quad \varepsilon_{t} \sim \mathcal{E S T}\left(0, \gamma_{\varepsilon}\right) \\
& \mu_{t}=\mu_{t-1}+\eta_{t} \quad, \quad \eta_{t} \sim \operatorname{RB}\left(0, \sigma_{\eta}^{2}\right)
\end{aligned}
$$

O componente sazonal determinístico $\omega_{t}$ é modelado com a parametrização trigonométrica, como em Harvey (1989):

$$
\omega_{t}=\sum_{j=1}^{6}\left(\omega_{c, j} \cos \frac{2 \pi j t}{12}+\omega_{s, j} \operatorname{sen} \frac{2 \pi j t}{12}\right)
$$

$\omega_{c, j}$ e $\omega_{s, j}, j=1, \ldots, 6$ são os parâmetros associados aos componentes cosseno e seno, respectivamente. Como sen $k \pi=0$ para $k$ inteiro, tem-se que $\omega_{s, 6} \equiv 0$ e, portanto, o número total de parâmetros é 11 , como na parametrização com dummies mensais. De fato, ambas parametrizações são equivalentes.

O teste de hipóteses, $\operatorname{com} \theta=\sigma_{\eta}^{2} / \sigma_{\varepsilon}^{2}, \quad \sigma_{\varepsilon}^{2}=\gamma_{\varepsilon}(0)>0, \quad$ é:

$$
\begin{aligned}
& H_{0}: \theta=0 \\
& H_{1}: \theta>0
\end{aligned}
$$

Os resíduos $\left\{e_{t}\right\}$ para o teste são obtidos pelo seguinte ajuste de mínimos quadrados:

$$
x_{t}=\hat{\mu}_{0}+\hat{\beta} t+\sum_{j=1}^{6}\left(\hat{\omega}_{c, j} \cos \frac{2 \pi j t}{12}+\hat{\omega}_{s, j} \operatorname{sen} \frac{2 \pi j t}{12}\right)+\epsilon_{t}
$$

A figura 6.3 mostra a reta ajustada e a série $\left\{x_{t}\right\}$ subtraída do componente sazonal ajustado. As estimativas dos parâmetros são

$$
\begin{aligned}
\hat{\mu}_{0} & =8.57 \\
\hat{\beta} & =0.00317 \\
\hat{\omega}_{c} & =(-0.0475,-0.0063,-0.0043,-0.0089,-0.0069,-0.0026) \\
\hat{\omega}_{s} & =(-0.0165,-0.0129,-0.0208,-0.0125,-0.0106,0)
\end{aligned}
$$


A estimativa de $\hat{\beta}$ corresponde a uma taxa de crescimento das exportações de $0.73 \%$ a.m. ou $9.15 \%$ a.a.

Pela metodologia apresentada no capítulo 3 , tem-se as seguintes estimativas para a largura de faixa ótima $l^{*}$ e a variância de longo termo $\sigma_{L}^{2}$ :

$$
\begin{aligned}
\widehat{l^{*}} & =28.3 \\
\hat{\sigma}_{L}^{2} & =0.572
\end{aligned}
$$

Chega-se então à estatística $\xi_{\beta}$ do teste ILO e ao p-valor associado a sua distribuição assintótica $\left(\sim \mathrm{CvM}_{2}\right)$ :

$$
\begin{aligned}
\xi_{\beta} & =0.262 \\
\text { p-valor } & =0.00375
\end{aligned}
$$

Ou seja, há forte evidência estatística para rejeitar a hipótese de estacionariedade em torno de uma tendência linear (trend stationarity) para a série de exportações analisada.

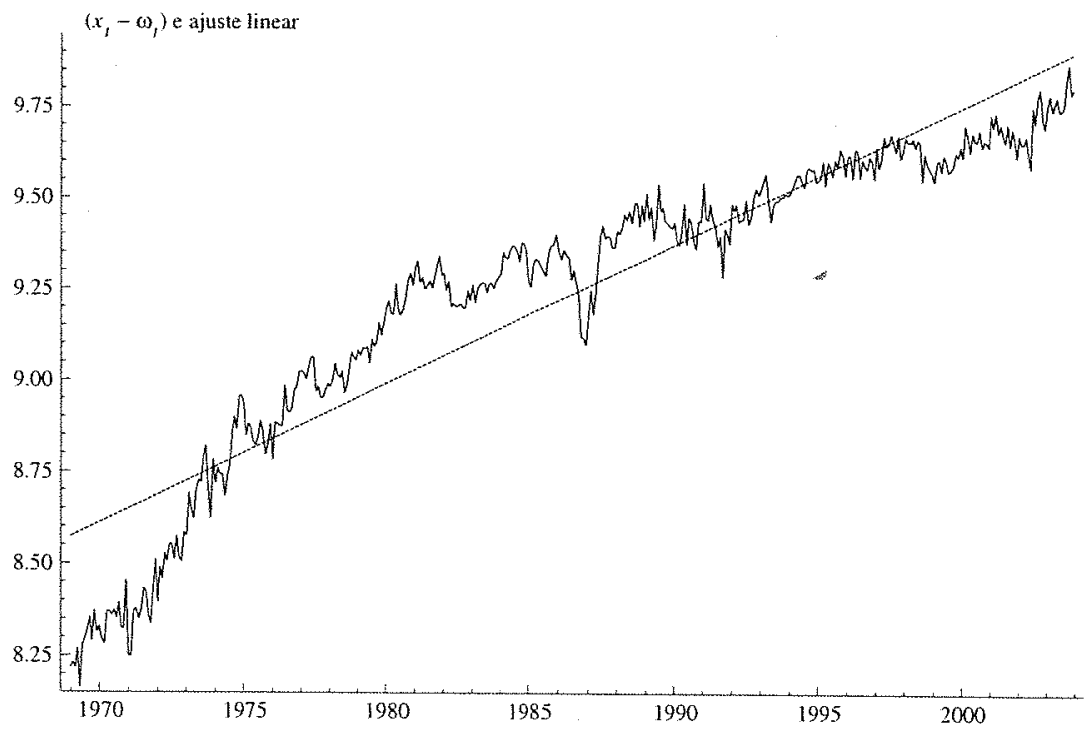

Figura 6.3: A série $x_{t}$ dessazonalizada e a reta ajustada 


\section{Modelo com tendência e com uma quebra estrutural}

Pela análise visual da série de exportações e pelo contexto histórico, conjecturamos uma quebra estrutural na série de exportações brasileiras em dezembro de 1981. Essa data pode ser identificada como o início de uma queda acentuada das reservas internacionais brasileiras devido às dificuldades de equilíbrio do balanço de pagamentos, decorrentes da crise da dívida externa no início da década de 1980. Essas dificuldades agravam-se ainda mais com a moratória mexicana em agosto de 1982 e, de certo modo, estendem-se até hoje devido à consequente redução da disponibilidade de crédito dos países desenvolvidos para os países em desenvolvimento.

Assim, introduzindo uma quebra estrutural no nível e na tendência em dezembro de 1981, tem-se o seguinte modelo

$$
\begin{aligned}
& x_{t}=\mu_{t}+\beta t+\delta_{\mu} w_{t}+\delta_{\beta}\left(w_{t} t\right)+\omega_{t}+\varepsilon_{t}, \quad \varepsilon_{t} \sim \mathcal{E S T}\left(0, \gamma_{\varepsilon}\right) \\
& \mu_{t}=\mu_{t-1}+\eta_{t}, \quad \eta_{t} \sim \operatorname{RB}\left(0, \sigma_{\eta}^{2}\right) \\
& \omega_{t}=\sum_{j=1}^{6}\left(\omega_{c, j} \cos \frac{2 \pi j t}{12}+\omega_{s, j} \operatorname{sen} \frac{2 \pi j t}{12}\right) \\
& w_{t}=\mathbf{1}_{\{t>156\}}, \quad \lambda=156 / 420=0.37
\end{aligned}
$$

O teste de hipóteses continua a ser como em (6.3) e os resíduos $\left\{e_{t}\right\}$ para o teste são obtidos pelo seguinte ajuste de mínimos quadrados:

$$
x_{t}=\hat{\mu}_{0}+\hat{\beta} t+\hat{\delta}_{\mu} w_{t}+\hat{\delta}_{\beta}\left(w_{t} t\right)+\hat{\omega}_{t}+e_{t}
$$


As estimativas dos parâmetros são :

$$
\begin{aligned}
& \hat{\mu}_{0}=8.24 \\
& \hat{\delta}_{\mu}=-0.125 \\
& \hat{\beta}=0.00713 \\
& \hat{\delta}_{\beta}=-0.00513 \\
& \hat{\omega}_{c}=(-0.0468,-0.0056,-0.0035,-0.0081,-0.0062,-0.0023) \\
& \hat{\omega}_{s}=(-0.0138,-0.0116,-0.0201,-0.0121,-0.0104,0)
\end{aligned}
$$

As estimativas das inclinações correspondem a um crescimento das exportações de $21 \%$ a.a. de janeiro/1969 a dezembro/1981 e de apenas $5.7 \%$ a.a. de janeiro/1982 a dezembro/2004. A figura 6.4 mostra as retas ajustadas e a série sem o componente sazonal ajustado.

As estimativas para a largura de faixa ótima $l^{*}$ e a variância de longo termo $\sigma_{L}^{2}$ são

$$
\begin{aligned}
\widehat{l^{*}} & =8.1 \\
\hat{\sigma}_{L}^{2} & =0.0211
\end{aligned}
$$

A estatística ${ }_{1} \xi_{\beta}$ do teste ILO e o p-valor associado a sua distribuição assintótica ( $\left.\lambda^{2} \mathrm{CvM}_{2}+(1-\lambda)^{2} \mathrm{CvM}_{2}\right)$ são

$$
\begin{aligned}
{ }_{1} \xi_{\beta} & =0.0544 \\
\text { p-valor } & =0.12305
\end{aligned}
$$

Ou seja, não se rejeita a hipótese de estacionariedade ao redor de uma tendência com quebra em dez/1981, até um nível de significância de $12 \%$. Portanto, tendo como plausível a conjectura de que de fato houve uma mudança estrutural no comportamento das exportações a partir de 1982, conclui-se que o ingresso de divisas, em US\$, advindas das exportações segue um processo estacionário ao redor de uma tendência determinística. 


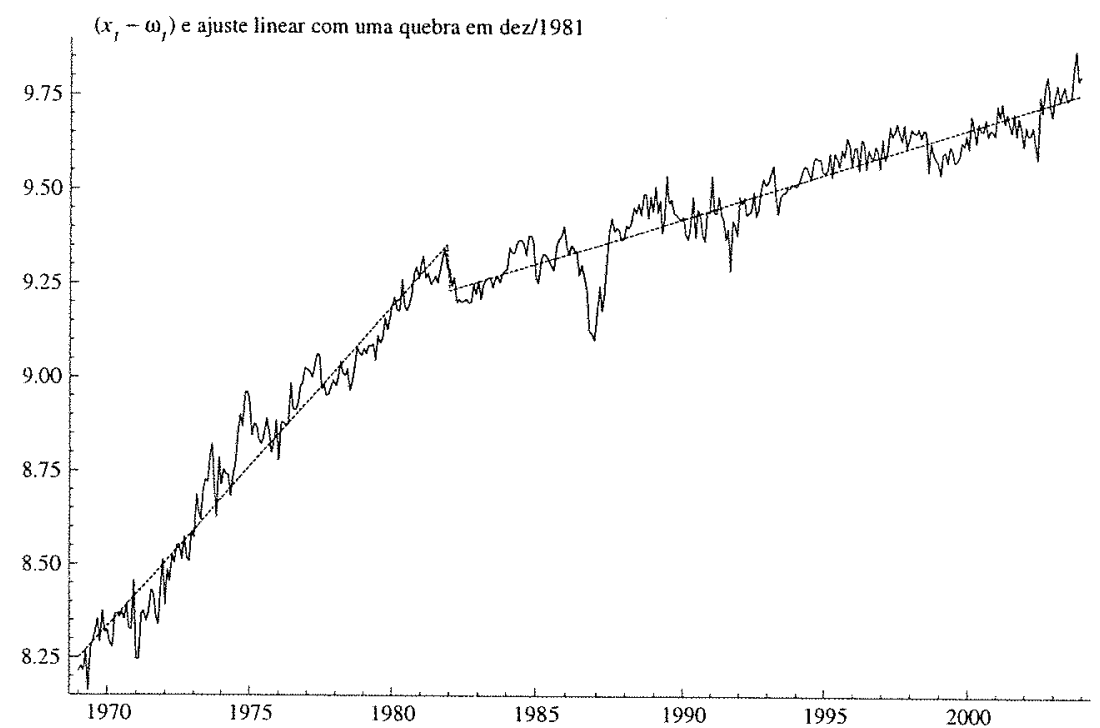

Figura 6.4: Série $x_{t}$ dessazonalizada e retas ajustadas com uma quebra simultânea no nível e na tendência em dezembro de 1981.

\subsection{Análise das importações brasileiras}

Analisamos aqui a série mensal de importações brasileiras, em dólar corrente, no mesmo período anterior, janeiro de 1969 a dezembro de 2003, para fins de comparação com o comportamento das exportações. Em princípio, as exportações e as importações de um país deveriam apresentar comportamento semelhante. Isso porque o equilíbrio na balança comercial é uma condição de solvência do país no longo prazo. Déficits sistemáticos na balança comercial encontram cada vez menos possibilidade de serem financiados via empréstimos ou investimentos externos.

Assim, espera-se que a série da balança comercial (exportações menos importações) seja estacionária, ou, no contexto de cointegração, que as séries de exportação e de importação sejam cointegradas. Nesse sentido é fundamental saber o comportamento de cada série individualmente, se são estacionárias, se apresentam mesma tendência, quebras etc. 


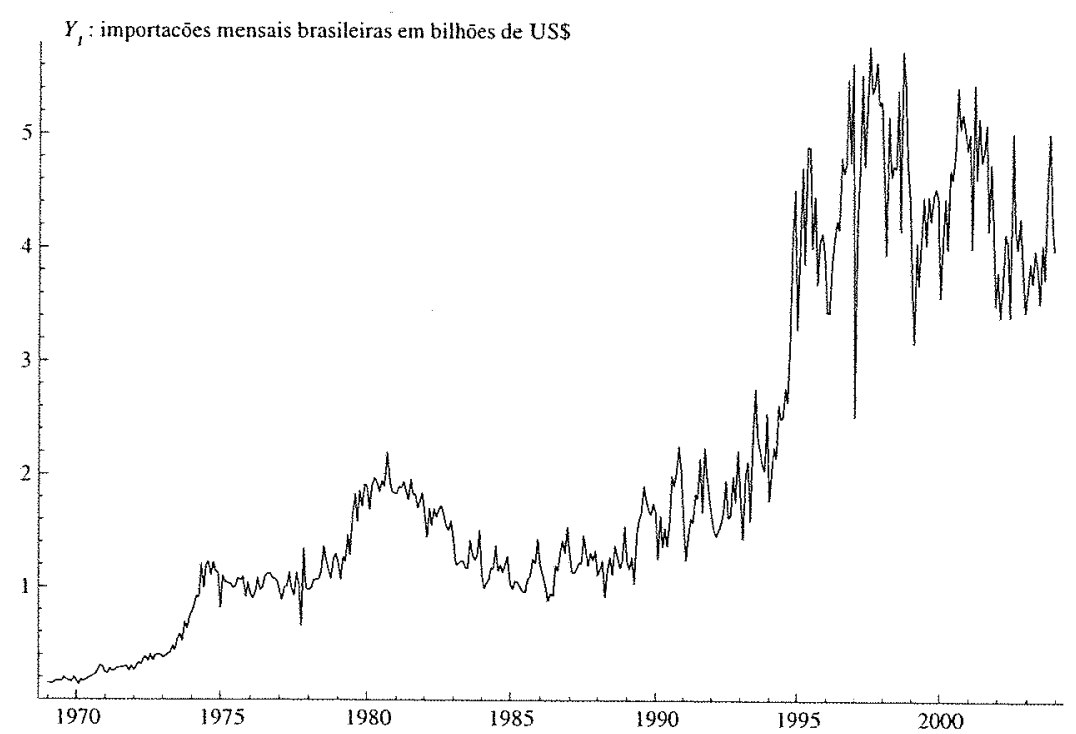

Figura 6.5: $Y_{t}=$ Importações mensais brasileiras, em bilhões de US\$, do período de janeiro/1969 a dezembro/2003, $t=1, \ldots, T=420$

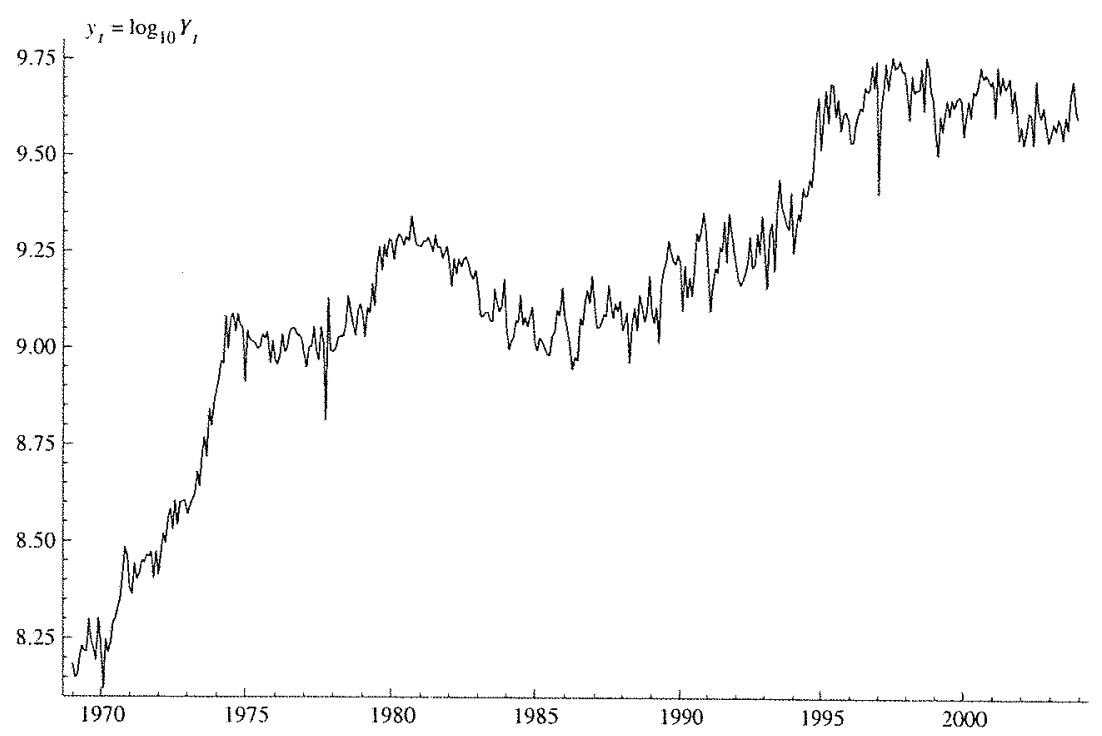

Figura 6.6: Logaritmo das importações mensais brasileiras, $y_{t}=\log _{10} Y_{t}$ 


\section{Modelo com tendência e sem quebras estruturais}

Novamente, o primeiro modelo testado é o com tendência, mas sem quebras estruturais:

$$
\begin{aligned}
y_{t} & =\mu_{t}+\beta t+\omega_{t}+\varepsilon_{t}, \quad \varepsilon_{t} \sim \mathcal{E S T}\left(0, \gamma_{\varepsilon}\right) \\
\mu_{t} & =\mu_{t-1}+\eta_{t}, \quad \eta_{t} \sim \operatorname{RB}\left(0, \sigma_{\eta}^{2}\right) \\
\omega_{t} & =\sum_{j=1}^{6}\left(\omega_{c, j} \cos \frac{2 \pi j t}{12}+\omega_{s, j} \operatorname{sen} \frac{2 \pi j t}{12}\right)
\end{aligned}
$$

E o mesmo teste de hipóteses

$$
\begin{aligned}
& H_{0}: \theta=0, \quad \theta \equiv \sigma_{\eta}^{2} / \sigma_{\varepsilon}^{2} \\
& H_{1}: \theta>0
\end{aligned}
$$

Os resíduos $\left\{e_{t}\right\}$ para o teste são obtidos pelo seguinte ajuste de mínimos quadrados:

$$
y_{t}=\hat{\mu}_{0}+\hat{\beta} t+\hat{\omega}_{t}+e_{t}
$$

A figura 6.7 mostra a reta ajustada e a série $y_{t}$ subtraída do componente sazonal ajustado. As estimativas dos parâmetros são

$$
\begin{aligned}
\hat{\mu}_{0} & =8.58 \\
\hat{\beta} & =0.00283 \\
\hat{\omega}_{c} & =(-0.0166,-0.0041,-0.0094,-0.0044,-0.0037,0.0000) \\
\hat{\omega}_{s} & =(-0.0278,-0.0100,-0.0128,-0.0052,-0.0132,0)
\end{aligned}
$$

A estimativa de $\hat{\beta}$ corresponde a uma taxa de crescimento das importações de $0.65 \%$ a.m. ou $8.14 \%$ a.a.

As estimativas para a largura de faixa ótima $l^{*}$ e a variância de longo termo $\sigma_{L}^{2}$ são

$$
\begin{aligned}
\widehat{l^{*}} & =23.9 \\
\hat{\sigma}_{L}^{2} & =0.734
\end{aligned}
$$




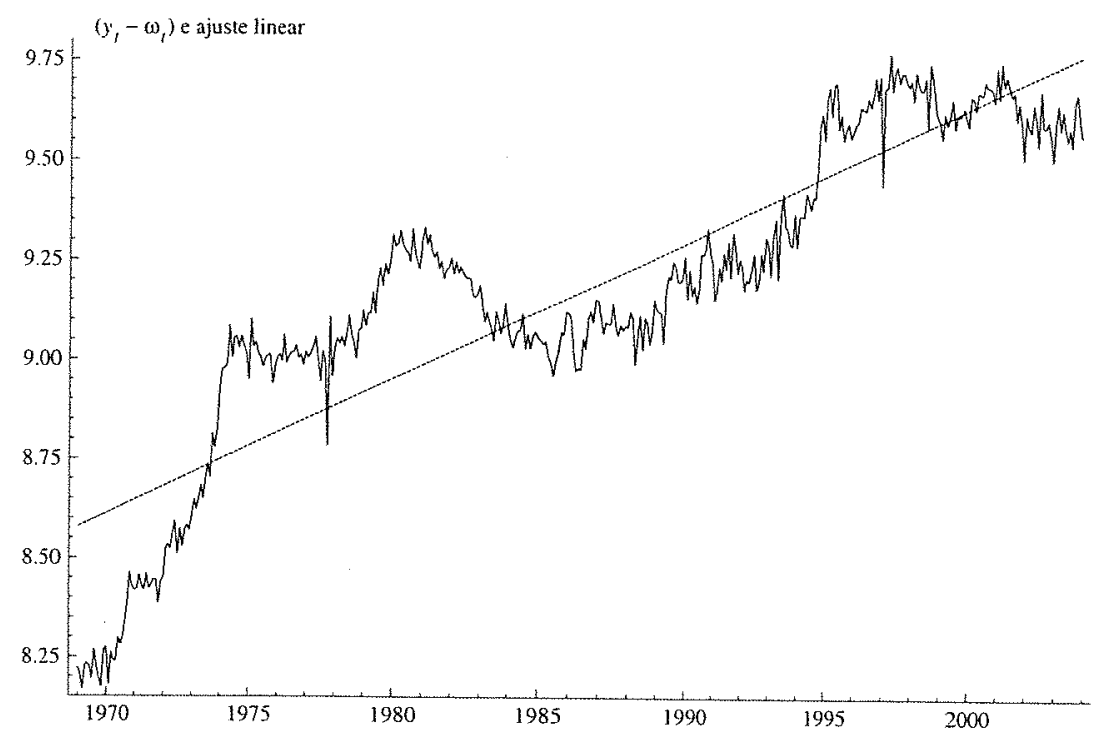

Figura 6.7: Série $y_{t}$ dessazonalizada e a reta ajustada

A estatística $\xi_{\beta}$ do teste $\| L O$ e o p-valor associado a sua distribuição assintótica ( $\mathrm{CvM}_{2}$ ) são

$$
\begin{aligned}
\xi_{\beta} & =0.127 \\
\text { p-valor } & =0.0821
\end{aligned}
$$

Ou seja, a hipótese de estacionariedade ao redor de uma tendência linear seria rejeitada a um nível de significância de $10 \%$, mas não seria a um um nível de significância de $5 \%$, por exemplo. Portanto, diferentemente da série de exportações, o teste não apresenta forte evidência de não-estacionariedade, podendo até ser considerada estacionária em torno de uma tendência, a um nível de significância de $5 \%$.

\section{Modelo com tendência e com uma quebra estrutural}

Pelo contexto histórico e pela análise visual da série de importações, identificamos preliminarmente quatro quebras estruturais plausiveis:

B1 : dez/1973 $(\tau=60)$, primeiro choque do petróleo. 
B2 : dez/1981 ( $\tau=156)$, crise da dívida externa.

B3 : $\operatorname{mar} / 1986(\tau=207)$, Plano Cruzado.

B4 : jul/1994 $(\tau=307)$, Plano Real.

Modelo

$$
\begin{aligned}
& y_{t}=\mu_{t}+\beta t+\delta_{\mu} w_{t}+\delta_{\beta}\left(w_{t} t\right)+\omega_{t}+\varepsilon_{t}, \quad \varepsilon_{t} \sim \mathcal{E S T}\left(0, \gamma_{\varepsilon}\right) \\
& \mu_{t}=\mu_{t-1}+\eta_{t}, \quad \eta_{t} \sim \operatorname{RB}\left(0, \sigma_{\eta}^{2}\right) \\
& \omega_{t}=\sum_{j=1}^{6}\left(\omega_{c, j} \cos \frac{2 \pi j t}{12}+\omega_{s, j} \operatorname{sen} \frac{2 \pi j t}{12}\right) \\
& w_{t}=\mathbf{1}_{\{t>\tau\}}, \quad \lambda=\tau / T
\end{aligned}
$$

Teste de hipóteses

$$
\begin{aligned}
& H_{0}: \theta=0, \quad \theta \equiv \sigma_{\eta}^{2} / \sigma_{\varepsilon}^{2} \\
& H_{1}: \theta>0
\end{aligned}
$$

\section{Resultados}

\begin{tabular}{cccccccc}
\hline \hline quebra & mês & $\tau$ & $\lambda$ & $\widehat{l}^{*}$ & $\hat{\sigma}_{L}^{2}$ & ${ }_{1} \xi_{\beta}$ & p-valor \\
\hline B1 & $12 / 73$ & 60 & 0.143 & 34.1 & 0.447 & 0.110 & 0.0507 \\
B2 & $12 / 81$ & 156 & 0.371 & 18.6 & 0.202 & 0.079 & 0.0278 \\
B3 & $03 / 86$ & 207 & 0.493 & 22.0 & 0.497 & 0.089 & 0.0057 \\
B4 & $07 / 94$ & 307 & 0.731 & 26.7 & 0.729 & 0.136 & 0.0056 \\
\hline
\end{tabular}

Nota-se que a introdução de apenas uma quebra simultânea no nível e na tendência, nas datas factíveis acima, conduz o teste no sentido de rejeição de $H_{0}$. 


\section{Modelo com tendência e mais de uma quebra estrutural}

Utilizando o teste simplificado da seção 4.3 e permitindo-se mais de uma quebra, nas mesmas datas acima, chega-se às seguintes combinações para as quais não se rejeita a hipótese de estacionariedade em torno de uma tendência com quebras simultâneas no nível e na tendência:

B12 : quebras em dez/1973 $\left(\tau_{1}=60\right)$ e dez/1981 $\left(\tau_{2}=156\right)$.

B14 : quebras em dez/1973 $\left(\tau_{1}=60\right)$ e jul/1994 $\left(\tau_{2}=307\right)$.

B134: quebras em dez/1973 $\left(\tau_{1}=60\right), \operatorname{mar} / 1986\left(\tau_{2}=207\right) \mathrm{e}$

$$
\mathrm{jul} / 1994\left(\tau_{3}=307\right) \text {. }
$$

\section{Resultados}

\begin{tabular}{cccccc}
\hline \hline quebras & $\widehat{l}^{*}$ & $\hat{\sigma}_{L}^{2}$ & ${ }_{2} \xi_{\beta}^{*}$ & ${ }_{3} \xi_{\beta}^{*}$ & p-valor \\
\hline B12 & 18.9 & 0.149 & 0.240 & - & 0.2418 \\
B14 & 24.8 & 0.109 & 0.184 & - & 0.5181 \\
B134 & 25.4 & 0.081 & - & 0.344 & 0.1631 \\
\hline
\end{tabular}

Das três combinações acima, destaca-se B14 que, com forte evidência estatística, não rejeita a hipótese de estacionariedade em torno de uma tendência com quebras em dez/1973 e jul/1994.

Uma possível interpretação sugerida é que os eventos que mais afetaram a série de importações brasileiras no período analisado foram a primeira crise do petróleo em 1973 e o Plano Real em 1994.

Importante destacar ainda que, pela metodologia utilizada, as séries de exportação e importação brasileiras apresentam comportamentos distintos, o que sugere uma maior dificuldade para se conseguir um equilíbrio na balança comercial. Aparentemente, as 
exportações são mais influenciadas pela disponibilidade de crédito externo e abertura aos mercados dos países desenvolvidos, ou seja, predominam os fatores externos. Já as importações são sensíveis tanto a choques externos (crise do petróleo, crise da dívida externa) como a choques resultantes da política econômica interna (Plano Real).

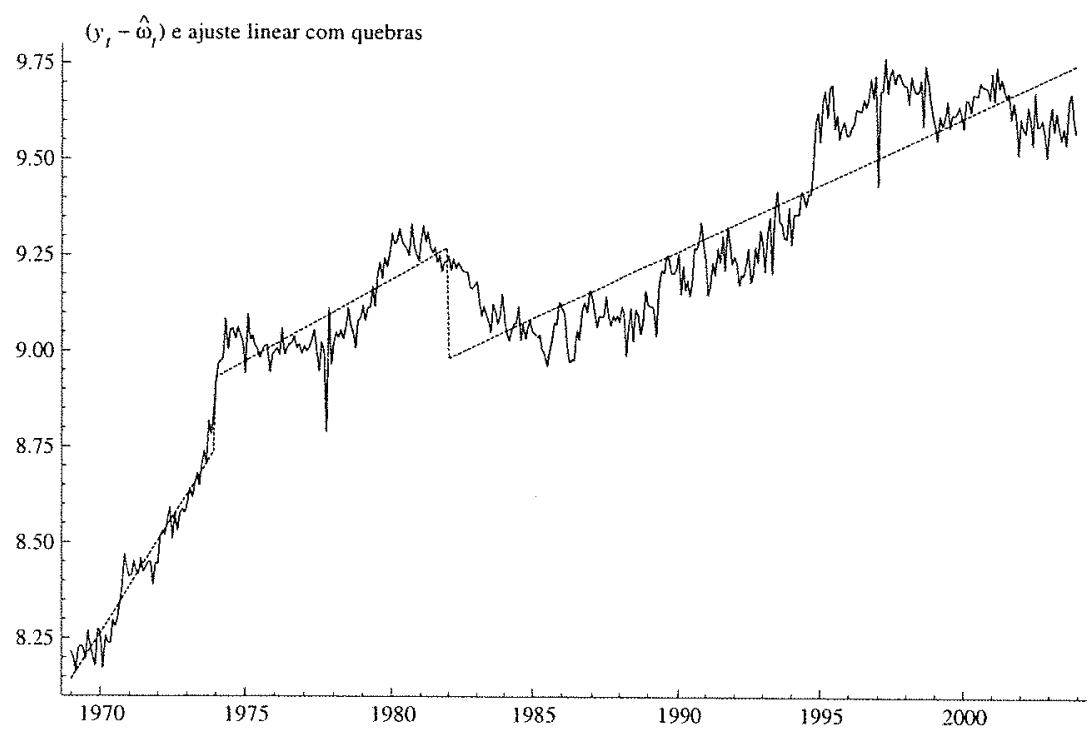

Figura 6.8: Série $y_{t}$ dessazonalizada e retas ajustadas com quebras em dez/73 e dez/81. 


\subsection{Análise das importações brasileiras}

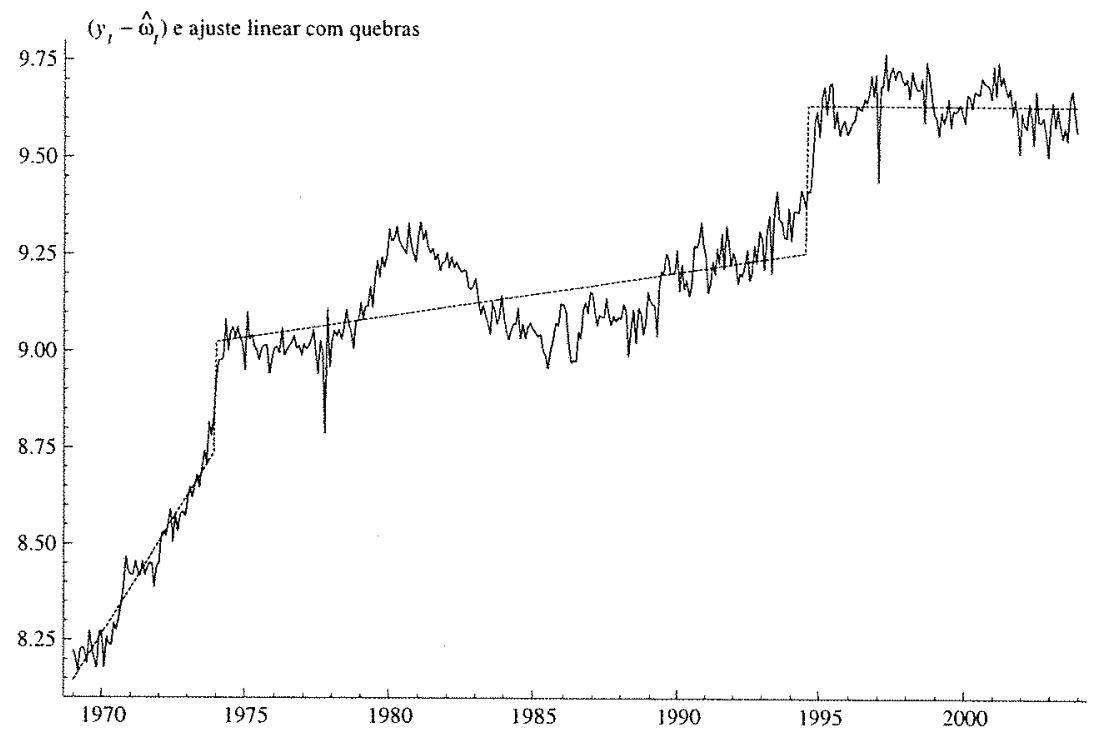

Figura 6.9: Série $y_{t}$ dessazonalizada e retas ajustadas com quebras em dez/73 e jul/94.

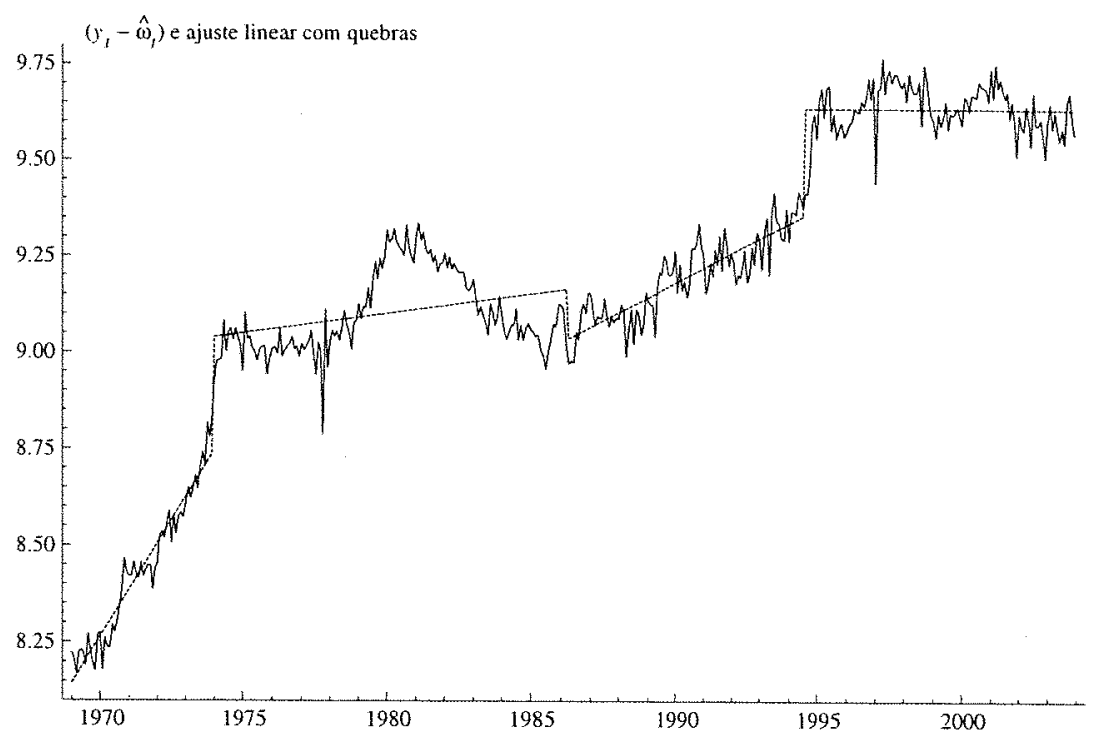

Figura 6.10: Série $y_{t}$ dessazonalizada e retas ajustadas com quebras em dez/73 e jul/94. 


\subsection{Câmbio real brasileiro}

A hipótese da paridade do poder de compra $(P P C)^{1}$, em sua forma relativa, estabelece que as variações da taxa de câmbio nominal $C$ acompanham, principalmente no longo prazo, as variações dos índices de preço doméstico $P_{d}$ e externo $P_{e}$. Posto de outra forma, espera-se que a taxa de câmbio real

$$
c=C \frac{P_{e}}{P_{d}}
$$

flutue em torno de um nível constante. Ou ainda, em termos estatísticos, que a série temporal de câmbio real $c_{t}$ seja estacionária.

A fim de testar a hipótese da PPC, utilizamos então a metodologia apresentada no capítulo 4 , no modelo sem tendência.

Para construir a série de câmbio real utilizou-se como índice de preço doméstico o IGP-DI geral mensal centrado de fim de período (fonte: IPEA), como índice de preço externo o IPC americano (fonte: FMI) e a taxa de câmbio nominal de venda de fim de mês (fonte: Banco Central do Brasil - Boletim de Balanço de Pagamentos). O período analisado é de janeiro de 1969 a dezembro de 2003.

A série construída encontra-se na figura 6.11 .

Modelo sem tendência e sem quebras

$$
\begin{aligned}
& c_{t}=\mu_{t}+\varepsilon_{t}, \quad \varepsilon_{t} \sim \mathcal{E S T}\left(0, \gamma_{\varepsilon}\right) \\
& \mu_{t}=\mu_{t-1}+\eta_{t}, \quad \eta_{t} \sim \operatorname{RB}\left(0, \sigma_{\eta}^{2}\right)
\end{aligned}
$$

\footnotetext{
${ }^{1}$ ver, por exemplo, Araújo \& Silveira (2002)
} 


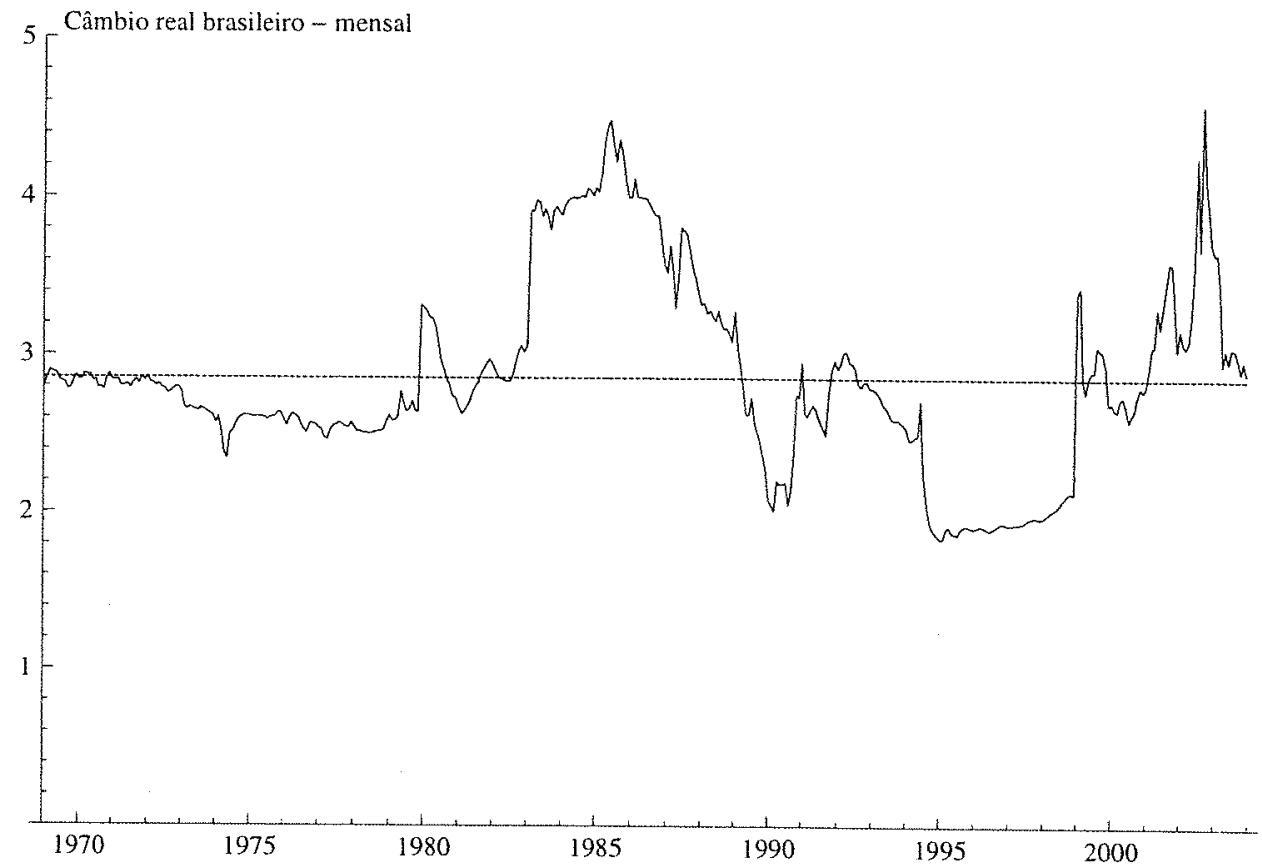

Figura 6.11: Taxa de câmbio real em R\$/US\$ - valor a cada final de mês de jan/1969 a dez/2003

Teste de hipóteses

$$
\begin{aligned}
& H_{0}: \theta=0, \quad \theta \equiv \sigma_{\eta}^{2} / \sigma_{\varepsilon}^{2} \\
& H_{1}: \theta>0
\end{aligned}
$$

\section{Resultados}

A estimativa para $\hat{\mu}_{0}$ é de 2.8535 e as estimativas para a largura de faixa ótima $l^{*}$ e a variância de longo termo $\sigma_{L}^{2}$ são

$$
\begin{aligned}
\hat{l^{*}} & =27 \\
\hat{\sigma}_{L}^{2} & =9.617
\end{aligned}
$$




\subsection{Câmbio real brasileiro}

A estatística $\xi_{\mu}$ do teste ILO e o p-valor associado a sua distribuição assintótica $(\sim \mathrm{CVM})$ são

$$
\begin{aligned}
\xi_{\mu} & =0.12473 \\
\text { p-valor } & =0.4766
\end{aligned}
$$

Portanto, não se rejeita a hipótese de estacionariedade para essa particular série de câmbio real brasileiro no período analisado. Assim, pela metodologia utilizada, há evidência estatística da validade da paridade do poder de compra. 


\section{Referências Bibliográficas}

[1] Anderson, T. W. e Darling, D. A. (1952) Asymptotic theory of certain "goodness of fit" criteria based on stochastic processes. Ann. Math. Stat., 23, 193-212.

[2] Andrews, D. J. (1991) Heteroskedasticity and autocorrelation consistent covariance matrix estimation. Econometrica, 59, 817-858.

[3] Araújo, C. H. V. e Silveira Fo, G. B. (2002) Mudanças de regime no câmbio brasileiro Working Paper Series - Banco Central do Brasil, WP41

[4] Billingsley, P. (1995) Probability and Measure 3a. edição. New York: WileyInterscience.

[5] Busetti, F. e Harvey, A. C. (2001) Testing for the presence of a random walk in series with structural breaks. J. Time Ser. Anal., 22, 127-150.

[6] Busetti, F. (2002) Testing for (common) stochastic trends in the presence of structural breaks. J. Forecast., 21, 81-105.

[7] Doornik, J. A. (1999) Object-Oriented Matrix Programming Using OX 3a, edição. London: Timberlake Consultants Press.

[8] Epanechnikov, V. A. (1969) Non-parametric estimation of multivariate probability density. Theory of Probability and Its Applications, 14, 153-158.

[9] Ferguson, T.S. (1967) Mathematical Statistics: A Decision Theoretic Approach. New York: Academic Press. 


\section{REFERÊNCIAS BIBLIOGRÁFICAS}

[10] Harvey, A. C. (1989) Forecasting, Structural Time Series Models and the Kalman Filter. Cambridge: Cambridge University Press.

[11] King, M. L. e Hillier, G. H. (1985) Locally best invariant tests of the error covariance matrix of the linear regression model. J. R. Statist. Soc. B, 47, 98-102.

[12] Kurozumi, E. (2002) Testing for stationarity with a break. J. Economet., 108, 63-99.

[13] Kwiatkowski, D., Phillips, P. C. B., Schmidt, P. e Shin, Y. (1992) Testing the null hypothesis of stationarity against the alternative of a unit root: How sure are we that economic time series have a unit root? J. Econometrics, 44, 159-178.

[14] Macneill, I. B. (1978) Properties of sequences of partial sums of polynomial regression residuals with applications to tests for change of regression at unknown times. Ann. Stat., 6, 422-433.

[15] Maddala, G. S. e Kim, I-M. (1998) Unit Roots, Cointegration, and Structural Change. Cambridge: Cambridge University Press.

[16] Nelson, C. R. e Plosser, C. I. (1982) Trends versus random walks in macroeconomic time series: some evidence and implications. J. Monet. Econ., 10, 139-162.

[17] Newey, W. K. e West, K. D. (1994) Automatic lag selection in covariance matrix estimation. Review of Economic Studies, 61, 631-653.

[18] Nyblom, J. (1986) Testing for deterministic linear trend in time series. J. Am. Stat. Assoc., 81, 545-549.

[19] Nyblom, J. e Makelainen, T. (1983) Comparisons of tests for the presence of random walk coefficients in a simple linear model. J. Am. Stat. Assoc., 78, 856-864.

[20] Parzen, E. (1957) On consistent estimates of the spectrum of a stationary time series. Ann. Math. Stat., 28, 329-348. 


\section{REFERÊNCIAS BIBLIOGRÁFICAS}

[21] Perron, P. (1989) The Great Crash, the oil price shock, and the unit root hypothesis. Econometrica, 57, 335-346.

[22] Phillips, P. C. B. (1987) Time series regression with a unit root. Econometrica, $55,277-301$.

[23] Phillips, P. C. B. e Perron, P. (1988) Testing for a unit root in time series regression. Biometrika, 75, 335-346.

[24] Piessens, R., Uberhuber, C. W. e Kahaner, D. K. (1983) QUADPACK, A Subroutine Package for Automatic Integration. Berlin: Springer-Verlag.

[25] Priestley, M. B. (1981) Spectral Analysis and Time Series. London: Academic Press.

[26] Sheather, S. J. e Jones, M. C. (1991) A reliable data-based bandwidth selection method for kernel density estimation. J. R. Statist. Soc. B, 53, 683-690.

[27] Shorack, G. R. e Wellner, J. A. (1986) Empirical Processes with Applications to Statistics. New York: John Wiley \& Sons.

[28] Tanaka, K. (1996) Time Series Analysis: Nonstationary and Noninvertible Distribuiton Theory. New York: Wiley-Interscience Publication. 